\title{
An invitation to Loop Quantum Gravity
}

\author{
Lee Smolin* \\ Perimeter Institute for Theoretical Physics, \\ 35 King Street North, Waterloo, Ontario N2J 2W9, Canada, and \\ Department of Physics, University of Waterloo, \\ Waterloo, Ontario N2L 3G1, Canada
}

February 1, 2008

\begin{abstract}
We describe the basic assumptions and key results of loop quantum gravity, which is a background independent approach to quantum gravity. The emphasis is on the basic physical principles and how one deduces predictions from them, at a level suitable for physicists in other areas such as string theory, cosmology, particle physics, astrophysics and condensed matter physics. No details are given, but references are provided to guide the interested reader to the literature. The present state of knowledge is summarized in a list of 42 key results on topics including the hamiltonian and path integral quantizations, coupling to matter, approaches to unification, extensions to supergravity and higher dimensional theories, as well as applications to black holes, cosmology and Plank scale phenomenology. We describe the near term prospects for observational tests of quantum theories of gravity and the expectations that loop quantum gravity may provide predictions for their outcomes. Finally, we provide answers to frequently asked questions and a list of key open problems.
\end{abstract}

To be submitted to Reviews of Modern Physics

*Email address:Ismolin@perimeterinstitute.ca 


\section{Contents}

1 Introduction $\quad 3$

2 The four basic observations $\quad 4$

3 The basic physical picture $\quad 7$

3.1 The basics of quantum spacetime . . . . . . . . . . . . . 7

3.2 Why loops? The kinematics of diffeomorphism invariant quantum gauge



3.3 Dynamics of constrained topological field theories . . . . . . . . . . . . 15

3.4 Horizons, black holes and boundaries . . . . . . . . . . . . . 18

3.5 The path integral formulation: spin foam . . . . . . . . . . . . . . 19

4 The main results of loop quantum gravity 21

4.1 The fundamental results of the canonical theory . . . . . . . . . . . . 21

4.2 Results on path integrals and spin foams . . . . . . . . . . . . 23

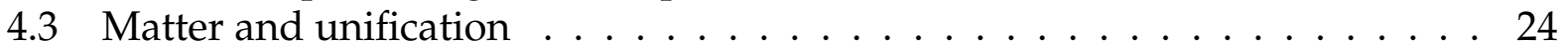

4.4 Results on black holes and horizons . . . . . . . . . . . . . . . 24

4.5 Results on the low energy behavior . . . . . . . . . . . . . . 26

4.6 Results concerning cosmology . . . . . . . . . . . . . . . 28

4.7 Results concerning supergravity and other dimensions . . . . . . . . . . . 29

4.8 Other extensions of the theory . . . . . . . . . . . . . . . . . . . . . . . . . . . . . .

4.9 Comments on the results . . . . . . . . . . . . . . . 30

5 The near term experimental situation 30

5.1 What does loop quantum gravity predict for the experiments? . . . . . . . 32

6 Frequently asked questions

7 Open questions in loop quantum gravity 40

8 Conclusions $\quad 42$ 


\section{Introduction}

Loop quantum gravity is a conservative approach to quantum gravity that asks a simple question: Can we construct a quantum theory of spacetime based only on the experimentally well confirmed principles of general relativity and quantum mechanics? Most approaches to quantum gravity have concluded or assumed that this could not be done. However, in the mid 1980 's the situation changed because it was understood that general relativity could be most simply formulated as a kind of gauge theory[1,2]. Given the tremendous advances in our understanding of gauge theories that had occurred in the decade previous, this made it possible to take a fresh approach to quantum gravity.

Remarkably, after almost two decades of work by a community of more than a hundred physicists and mathematicians, a great deal of evidence has accumulated that the answer to the question above is yes. The result is a language and dynamical framework for studying the physics of quantum spacetimes, which is completely consistent with the principles of both general relativity and quantum field theory. The picture of quantum spacetime geometry which emerges is to many compelling, independently of the fact that it has been derived from a rigorous quantization of general relativity. The basic structure that emerges is of a new class of quantum gauge field theories, which are background independent, in that no fixed spacetime metric is needed to describe their quantum dynamics[4]-[15].

While a number of issues remain open, enough is known about the physics of loop quantum gravity that a number of applications to problems of physical interest are under development. There is by now a well understood detailed description of the quantum physics of black hole and cosmological horizons $[16,17,18]$ that reproduces the BekensteinHawking results on the relationship between area and entropy[19]. There is under development an approach to quantum cosmology that shows that cosmological singularities are eliminated[20] and has already led to some predictions for effects observable in CMB spectra[20, 21]. And there are published predictions for observable Planck scale deviations from energy momentum relations[22, 23] that imply predictions for experiments in progress such as AUGER and GLAST. For those whose interest is towards speculations concerning supersymmetry and higher dimensions, there are also results that show how the methods of loop quantum gravity may be extended to give background independent descriptions of quantum gravity in the higher and super realms[32]-[36]. It thus seems like a good time for an introduction to the whole approach that may help to make the basic ideas, results and methods accessible to a wider range of physicists.

The main purpose of this review is to explain how it has been possible to obtain exact results about quantum gravity, describe the main features of the resulting theory, and then to list its basic results. The text is aimed at physicists who are not specialists in quantum gravity, particularly those in elementary particle physics, string theory, cosmology, high energy astrophysics and condensed matter physics. I assume only that the reader if familiar with the basics of gauge fields, quantum theory and general relativity.

Like any mature field, work in loop quantum gravity proceeds through a variety of 
methods and levels of rigor. The first results were found using direct extensions of methods from ordinary gauge theories, with modifications in regularization methods appropriate to theories without background metrics. More recently, all the key results have been confirmed using mathematically rigorous methods[7, 8]. In this review I employ a non-rigorous style, grounded on intuitions about the behavior of gauge fields that particle physicists and condensed matter physicists will find familiar. But the reader should keep in mind that, as will be noted, many of the key results listed are confirmed by completely rigorous methods. This is good, as we have so far no experimental checks on the behavior of gauge fields in this context, it is essential to be able to confirm our intuitions with rigorous results.

In the next section we describe the four basic observations which lead to loop quantum gravity. This is followed by an intuitive description of the basic physical picture. In section 4 we give a list of 42 key results achieved so far concerning the theory. We then summarize the near term experimental situation for loop quantum gravity and other theories in section 5. Section 6 gives answers to a list of questions most frequently asked by those curious or skeptical about loop quantum gravity, after which we close with a list of open problems.

For those interested in a more detailed introduction to loop quantum gravity, there are several textbooks and monographs available ${ }^{1}$ Review papers ${ }^{2}$ on different aspects of loop quantum gravity include [8]-[15].

\section{The four basic observations}

While the principles assumed are only those of general relativity and quantum mechanics, there are four key observations that make the success of loop quantum gravity possible. These are

I. Classical general relativity is a background independent theory, hence any theory which is to have general relativity as a low energy limit must be background independent. A background independent theory is one whose formulation does not assume or require the existence of any single preferred spacetime metric or connection. Instead, all the fields that define the geometry of spacetime are fully dynamical, none are fixed ${ }^{3}$.

The argument that a quantum theory of gravity must be background independent can be found developed in several places[6,128], where one can also find the term carefully

\footnotetext{
${ }^{1}$ Early monographs are $[4,5]$. A recent textbook by Rovelli[6] is from Cambridge University Press, with an early draft available on line at http://www.cpt.univ-mrs.fr rovelli/rovelli.html. Comprehensive introductions to the rigorous side of the subject are given in [7], also soon to appear from CUP, as well as the very recent review[8].

${ }^{2}$ References here are not intended to be complete, and are meant only as an introduction to some key results in the literature. Reminders of references I have forgotten will be appreciated.

${ }^{3}$ The reader should be aware that the word "background independence" has other connotations besides that given here. More about this as an answer to a FAQ.
} 
defined.

The principle of background independence does not imply that the fundamental theory must be based on fields living on a manifold. But it does imply that whenever manifolds appear, whether fundamentally or as part of the low energy effective theory, the dynamics of the fields will be invariant under active diffeomorphisms of the manifold. The reason for this was worked out first in detail by Dirac, and his [25] is still a good source for understanding the connection between background independence and diffeomorphism invariance ${ }^{4}$.

When we say that LQG realizes the basic principles of general relativity, we are referring to only the big principles, which include background independence, diffeomorphism invariance and the equivalence principle. While many papers in LQG are concerned with the quantization of the Einstein action, we can equally well study other actions, including supergravity and terms of higher powers in curvature. Loop quantum gravity is perfectly compatible with the expectation that the Einstein equations are just the low energy limit of a more fundamental theory.

II. Duality and diffeomorphism invariance may be consistently combined in a quantum theory. By duality we mean here the conjecture that the dynamics of a quantum gauge field can be described equivalently in terms of the dynamics of one dimensional extended objects. In the context of Yang-Mills theory this conjecture was explored in detail by Polyakov, Migdal, Mandelstam, Nielson and others, where the one dimensional objects were Wilson loops. More recently, in the background dependent context, the idea of duality between gauge fields and extended objects has been central string theory, for example in the $A d S / C F T$ correspondence. In the background independent context, the idea is central to loop quantum gravity. As we will see, the key point is a method for constructing a diffeomorphism invariant quantization of any diffeomorphism invariant gauge field theory. This cannot be done using Fock states, as the inner product on Fock space depends on a background metric, whose presence breaks diffeomorphism invariance. But, as I will describe, it can be done if one works in a space of states created by the action of Wilson loops.

Indeed, from a technical point of view, the main achievement of loop quantum gravity is the discovery of a new kind of quantum gauge field theory, which is exactly invariant under diffeomorphisms of a manifold. This is understood in a great deal of detail, and the Hilbert space, inner product, states, observables and path integral representation are all understood in closed form. That understanding is robust, and has been achieved by several regularization procedures. Significantly, there is a completely rigorous formulation of these diffeomorphism invariant gauge theories. A recent uniqueness theorem[31] greatly limits the possibilities for quantum descriptions of diffeomorphism invariant gauge theories apart from the one studied in LQG.

III. General relativity and all related theories, such as supergravity, can be formulated as gauge

\footnotetext{
${ }^{4}$ This is also described in detail in [6] and in [26]-[30].
} 
theories. This means that the configuration variable is a gauge field, and the metric information is contained in the conjugate momenta to the gauge field. This extends to coupling with all the known kinds of matter fields.

IV. Further, general relativity, supergravity and related theories can be put in a special form in which they are constrained topological field theories. These are defined in section 3.3, briefly, they are theories whose actions differ from the action of a topological field theory ${ }^{5}$ by the imposition of a non-derivative, quadratic constraint equation. That constraint diminishes the number of gauge invariances, leading to the emergence of local physical degrees of freedom, while maintaining the diffeomorphism invariance of the theory. This is true of general relativity in all dimensions and is also known to be true of supergravity, in $d=4$, at least up to $N=2$ [32]-[34], and for $d=11$ [35].

As a result, the techniques which give us consistent diffeomorphism invariant gauge theories can be applied to give consistent quantizations of general relativity and supergravity. Many results follow, which will be described in more detail below. Most of the key results, have been confirmed as theorems in the rigorous formulation of diffeomorphism invariant quantum field theory.

The fundamental result which follows from these four observations is that quantum geometry is discrete 6 . Operators which measure the areas and volumes of diffeomorphism invariantly defined surfaces and regions may be constructed. They are finite, after an appropriate regularization, respecting diffeomorphism invariance, and they have discrete, computable spectra. Hence, the theory predicts minimal physical areas and volumes. By diagonalizing these observables we find an orthonormal basis of diffeomorphism invariant states, which are certain labeled graphs called spin networks. Their evolution can be described in closed form in either a Hamiltonian or path integral language. Furthermore, the dynamics of the matter fields can be constructed and studied. Because of the existence of minimal quanta of volume, all divergences of ordinary quantum field theories are eliminated. This is because there simply are no degrees of freedom in the exact theory that correspond to gravitons or other quanta with wavelength shorter than the Planck length. There is evidence as well that singularities of general relativity are eliminated.

It should be apparent from this summary that loop quantum gravity, as a research program, is rather different from other research programs such as string theory, that are based on new hypotheses about nature, such as supersymmetry, the existence of higher dimensions, and the unification of all elementary particles and forces by means of strings. These are interesting hypotheses, but it is proper to characterize them as speculative, as they have no direct support from experiment. Nor are there results that show that any of these assumptions are necessary consequences of a consistent unification of quantum theory with gravity and spacetime. It is fair to say that approaches such as string theory

\footnotetext{
${ }^{5} \mathrm{~A}$ topological field theory is a field theory whose equations of motion are all trivial, so that there are no local degrees of freedom. The solutions are parameterized instead entirely by topological and boundary information.

${ }^{6}$ Precise statements and references for all these results are given in section 4 .
} 
have so far failed to lead to a complete and well defined theory ${ }^{7}$. Nor have they led to many results that concern the behavior of quantum spacetime at the Planck scale. There is a widely held view that, to give a deeper picture of quantum spacetime, string (or perhaps $\mathcal{M}$ ) theory require a background independent formulation. It is natural to try to construct such a theory using the methods of loop quantum gravity, some preliminary results in this direction are given in $[35,36]$.

There are several different approaches to a background independent quantum theory of gravity. Besides loop quantum gravity, others include dynamical triangulations[38, 39], causal sets[37] and the Gambini-Pullin discrete quantization approach to quantum gravity[40]. Although these are independently motivated research programs, some of their results are relevant for loop quantum gravity, because they concern models which can be understood as arising from spin foam models by simplifications which eliminate certain structures.

It is also the case that while most results concern quantum general relativity, the methods of loop quantum gravity can be applied to construct a large number of different background independent quantum theories, and it can easily incorporate speculative hypotheses about the dimension of spacetime, the form of the action or the presence of additional symmetries such as supersymmetry. All of these theories incorporate the basic principles of background independence and diffeomorphism invariance, so that the spacetime geometry is completely dynamical.

At the same time, by showing that a consistent diffeomorphism invariant quantum field theory can be constructed that represents general relativity, coupled to arbitrary matter fields, in the $3+1$ dimensional world we observe, the success of loop quantum gravity undermines the claim that supersymmetry, strings or higher dimensions are necessary for a consistent quantum theory of gravity.

\section{The basic physical picture}

Before listing the main results of the program, I provide here a simple physical picture of how quantum spacetime is understood in loop quantum gravity. The picture is simple and intuitive, and I will present it without the calculations and theorems that back it up. The reader should be aware that there is a completely rigorous mathematical formulation supporting this picture, described in references to the results listed in the next section.

\subsection{The basics of quantum spacetime}

We begin with the basic picture, which is very simple to state. To define the kinematics of a loop quantum gravity theory, pick one from each of the following

- A topological manifold, $\Sigma$, say $S^{3}$.

\footnotetext{
${ }^{7} \mathrm{~A}$ detailed list of results from string theory is contained in [12].
} 
- A Lie algebra or, more generally, a Hopf algebra or superalgebra, $\mathcal{A}$, say $S U(2)$.

An $\mathcal{A}$-spin network, $\Gamma$, is a graph, whose edges are labeled by representations of $\mathcal{A}$. Each node is labeled by an invariant in the product of the representations of the incident edges.

Let $\{\Gamma\}$ be an embedding of the graph $\Gamma$ into the manifold $\Sigma$, up to topology. Then the Hilbert space $\mathcal{H}$ of the theory is defined by the following statement

$\mathcal{H}$ has an orthonormal basis $\mid\{\Gamma\}>$ labeled by the embeddings of the spin networks in the manifold $\Sigma$.

The states are interpreted in terms of operators which measure geometric properties such as the volumes and areas of regions and surfaces in $\Sigma$. Roughly speaking, the spin network states, $\mid\{\Gamma\}>$, are eigenstates of these observables. Each node of a spin network contributes a quanta of volume to any region which contains it, which is finite in Planck units and a function of the labelings. Each edge similarly contributes a quanta of area to any surface it crosses.

There are a number of variants, depending on the specification of $\Sigma$. We may specify that $\Sigma$ is a differential manifold, in which case the classes $\{\Gamma\}$ are equivalent up to diffeomorphisms (or, in some formulations, piecewise diffeomorphisms) of $\Sigma$. If $\Sigma$ has a boundary, that can indicate an asymptotic region, or a horizon of a black hole. Or one can drop $\Sigma$ and build the theory just from combinatorial graphs.

The dynamics is given in terms of the basis states as follows. Consider a small number of local moves, by which one or a few connected nodes change. Give each an amplitude, which is a function of the labels involved.

Then, a quantum history from an initial basis state to a final basis state is a succession of such moves. Each history has an amplitude which is a product of the amplitudes of each move. The quantum theory can then be defined by a sum over histories.

Each such history may be considered a quantum version of a spacetime. It has a discrete causal structure, because the moves can be given a partial order which indicate that a later move acted on a region which resulted from a previous move.

\subsection{Why loops? The kinematics of diffeomorphism invariant quantum gauge theories}

Where does the picture I just sketched come from? It has been derived from a new kind of quantum gauge theory which is diffeomorphism invariant. This construction has been successfully applied to a wide variety of theories, including topological field theories, general relativity and supergravity. It works in any dimension and all the standard kinds of matter fields can be included. The basic ideas behind this construction are very simple, from a physics point of view, although the math needed to realize them rigorously is a bit more involved. 
We begin by considering a theory of a connection ${ }^{8}, A_{a}^{i}$, valued in a Lie algebra (or superalgebra) $\mathcal{A}$ on a $d+1$ dimensional spacetime manifold $\mathcal{M}$. The manifold has no fixed metric defined on it, fixed or dynamical, all that is fixed is the topology and differential structure of $\mathcal{M}$. To quantize a spacetime theory using Hamiltonian methods, we assume that $\mathcal{M}=\Sigma \times R$, where $\Sigma$ proscribes the topology and differential structure of what we will call "space".

We begin with the Hamiltonian theory. The initial configuration space $\mathcal{C}$ will consist of the possible configurations of the gauge field $A_{a}^{i}$ on $\Sigma$. To this must be added the conjugate momenta, which are represented by the electric field $\tilde{E}_{i}^{a}$, which is a vector density on $\Sigma$, valued in the lie algebra $G$. Together, they coordinatize the phase space, on which is defined the Poisson bracket relations,

$$
\left\{A_{a}^{i}(x), \tilde{E}_{j}^{b}(y)\right\}=\delta^{3}(x, y) \delta_{a}^{b} \delta_{j}^{i}
$$

We note that because $\tilde{E}_{i}^{a}$ is a density this is well defined in the absence of a background metric.

The physical configuration space consists of equivalence classes of $\mathcal{C}$ under the action of the gauge symmetries of the theory. These include ordinary gauge transformations, with gauge group $\mathcal{A}(\Sigma)$ and the diffeomorphisms of $\Sigma$, denoted, $\operatorname{Diff}(\Sigma)$.

The physical configuration space we are interested in is then,

$$
\mathcal{C}^{\text {diffeo }}=\frac{\text { A- connections on } \Sigma}{\mathcal{A}(\Sigma) \times \operatorname{Diff}(\Sigma)}
$$

The problem we want to solve is how to write a corresponding Hilbert space of gauge and diffeomorphism invariant states. We proceed in three steps.

1. We construct a Hilbert space of gauge invariant states, called $\mathcal{H}^{\text {kin }}$, on which the diffeomorphisms of $\Sigma$ act unitarily and without anomalies.

2. We mod out by the unitary action of the diffeomorphisms, to find the subspace

$$
\mathcal{H}^{\text {diffeo }} \subset \mathcal{H}^{\text {kin }}
$$

of diffeomorphism invariant states.

3. We endow $\mathcal{H}^{\text {diffeo }}$ with an inner product, making it a Hilbert space.

For the first step, we cannot take the Fock space, which is the usual starting point for quantization of field theories. The reason is that Fock space depends on a background metric, and this prevents the construction of a unitary, anomaly free realization of the diffeomorphism group.

\footnotetext{
${ }^{8}$ All fields described here are forms, the form indices are $a=1, \ldots, d$, while the indices $i, j \ldots$ are valued in the Lie algebra, $\mathcal{A}$
} 
How do we find an alternative to Fock space, on which we can construct a unitary, anomaly free action of the diffeomorphisms? The key point is that there is no way to do this based on a quantization of the canonical commutation relations (1). Instead we start with a different set of Poisson bracket relations, made by certain extended objects.

An interesting fact about non-Abelian gauge theories is that you cannot coordinatize the space of connections, mod gauge transformations, completely in terms of $A_{a}^{i}$ and its derivatives at a point[24]. To label all distinct gauge invariant configurations you have to give the values of the holonomies. These are defined as follows. Given a path $\alpha \in \Sigma$ the path ordered exponential is defined as

$$
U[\alpha]=P e^{\int_{\alpha} A}
$$

Here $A=A^{i} \tau_{i}$, where $\tau_{i}$ is a generator of $\mathcal{A}$ in the fundamental representation.

The holonomy is the path ordered exponential around a closed loop, $\gamma$

$$
h[\gamma, A]=P e^{\int_{\gamma} A}
$$

This transforms under gauge transformations acting at the beginning of the loop. A gauge invariant observable is the Wilson loop, which is the trace of an holonomy,

$$
T[\gamma, A]=\operatorname{Tr} P e^{\int_{\gamma} A}
$$

To define a conjugate variable, let us for the moment fix $d=3$. The electric field, as a density is equivalent to a 2 -form

$$
E_{a b}^{i}=\epsilon_{a b c} \tilde{E}^{c i}
$$

This can be integrated against any surface $S \in \Sigma$ to yield the electric flux through $S$,

$$
E(S)^{i}=\int_{S} d^{2} S^{a b} E_{a b}^{i}
$$

These have a closed algebra under Poisson brackets

$$
\left\{U[\alpha, A], E[S]^{i}\right\}=\operatorname{Int}[\alpha, S] U\left[\alpha_{1}, A\right] \tau^{i} U\left[\alpha_{2}, A\right]
$$

where $\operatorname{Int}[\alpha, S]$ is the intersection number of the surface and path, $\alpha_{1}$ is the path from the origin to the point of intersection, and $\alpha_{2}$ is the rest of the path.

We can now state the key theorems of the subject:

- Existence: There exists a representation of (9) which carries a unitary and anomalyfree representation of the group of diffeomorphisms of $\Sigma$.

\footnotetext{
${ }^{9}$ The construction we are about to sketch works for all $d \geq 2$.
} 
- Uniqueness: This representation is unique[31] ${ }^{10}$.

We then take for $\mathcal{H}^{k i n}$ this unique representation.

The mathematics involved to prove these theorems is rather involved. But the physical picture behind them is very intuitive, and familiar to physicists. It is in fact the old idea of duality in Yang-Mills theories:

The vacuum is a dual superconductor, so that the electric flux measured by $E[S]$ is quantized. The physical excitations of this vacuum are defined by Wilson loops acting to create normalizable states, which are states of quantized non-Abelian electric flux.

It is easy to give an intuitive picture of how the unique representation $\mathcal{H}^{\text {kin }}$ is built.

We may begin with a vacuum state $\mid 0>$ which is an eigenstate of electric flux with vanishing flux everywhere.

$$
E[S]^{i} \mid 0>=0
$$

for all surfaces $S$. We then create a state which has one unit of non-abelian electric flux around a loop $\gamma$ by acting on the vacuum with a Wilson loop operator.

$$
\hat{T}[\gamma, A]|0>=| \gamma>
$$

It pays to work with a gauge invariant version of the electric flux

$$
\mathcal{E}[S]=\int_{S} \sqrt{E^{i} E^{i}}
$$

It turns out that this operator can be defined through a regularization procedure, without breaking diffeomorphism invariance, and the result is a finite operator.

The state with one loop excited is an eigenstate of $\mathcal{E}[S]$

$$
\mathcal{E}[S]|\gamma>=\hbar \operatorname{Int}[\gamma, S]| \gamma>
$$

If we write the dependence on the connection $A_{a}^{i}$ then the state created by the Wilson loop is just

$$
<A \mid \gamma>=T[\gamma, A]
$$

Given a set of loops $\gamma_{i}, i=1, \ldots M$, for $M$ finite, we can build up complicated states

$$
\prod_{i} \hat{T}\left[\gamma_{i}, A\right]|0>=|\left\{\gamma_{i}\right\}>
$$

Its value is

$$
<A \mid\left\{\gamma_{i}\right\}>=\prod_{i} T\left[\gamma_{i}, A\right]
$$

Such states are not normalizable in Fock space. But this is of little concern for us, as there can be no Fock space in the absence of a background metric. It is also the case that

\footnotetext{
${ }^{10}$ Modulo some technical assumptions, described below and in [31]
} 
there are no operators in the theory that represents $A_{a}^{i}(x)$ or their field strengths $F_{a b}^{i}(x)$. The gauge field is represented only by the non-local operators $\hat{T}[\gamma, A]$, but the limits for very small loops, which classically would give the field strengths, do not exist.

In a certain sense, this representation is closer to the Hilbert spaces of lattice gauge theory than to Fock space. Of course, a lattice is a background structure, and we don't use a fixed lattice here, we simply consider all such states. The new representation can be thought of roughly as the direct sum of the Hilbert spaces of all lattice gauge theories, with all possible lattices. This indeed is how the construction is done in the rigorous approach.

The key idea is that quantum geometries are built up from such states. There is a translations between gauge fields and gravity, which follows from observations III and IV above. In $3+1$ dimensions the correspondence gives $\mathcal{A}=S U(2)$ or $S O(3)^{11}$ which we will assume for the following. This gives us correspondences between flux of the electric field $\tilde{E}_{i}^{a}$ and geometric quantities. Given a surface $S$, it turns out that

$$
\text { Area of } S=\hbar G \mathcal{E}[S]
$$

Hence the states created by the Wilson loops acting on the vacuum $\mid 0>$ are eigenstates of the operators that measure the areas of surfaces $S$. So it follows that the dual superconductor picture leads to a quantization of areas.

Note the $\hbar G$ in (17). This is necessary because area and electric flux have different dimensions. When the correspondence is worked out in detail, they are there because $\hbar$ and $G$ are parameters of a quantum theory of gravity. Because of them, flux quanta turn into quanta of area, with a minimal quanta of area given by the Planck area $\hbar G$.

Further, given a region $R \in \Sigma$ the volume of $R$ can be expressed as

$$
\text { Volume of } R=(\hbar G)^{\frac{3}{2}} \int_{R} \sqrt{\left|\operatorname{det}\left(\tilde{E}_{i}^{a}\right)\right|}
$$

It is then of interest to construct simultaneous eigenstates of the operators that measure the volumes of all regions $R \in \Sigma$, and the areas of all surfaces that separate the regions. This is done by combining loops into the spin network graphs introduced above. This is necessary because it can be shown that after suitable regularization, the operator corresponding to (18) annihilates states of the form of (15) unless there are points where at least two loops intersect. Evidently, volume is a property associated with intersections of loops. The eigenstates turn out to be spin network states.

To give the definition of spin network states we return to general $\mathcal{A}$. A spin network $\Gamma$ is a graph whose edges are labeled with irreducible representations of $\mathcal{A}$. The nodes are also labeled by invariants (or intertwiners). If a node $n$ has edges incident on it with labels $i, j, k, l$, than the node has to be labeled by an invariant, defined by a map,

$$
\mu: i \otimes j \otimes k \otimes l \rightarrow I d
$$

\footnotetext{
${ }^{11}$ For $N=1$ supergravity, $G=O \operatorname{sp}(1,2)$.
} 
For each set of labels on the incident edges there is a finite dimensional space of such invariants. We require that there be a non-trivial such invariant at each node.

Given such a spin network $\Gamma$ we can define a spin network state, $\mid \Gamma>$. One way to define it is in terms of connections

$$
T[\Gamma, A]=<A \mid \Gamma>
$$

is a generalization of a Wilson loop. It is gotten by writing the parallel transports of the gauge field $A$ for each edge, in the representation labeling that edge, and then tracing them together, using the invariants labeling each node, to get a gauge invariant functional of the connection associated to the whole graph.

We can also write a spin network state as a sum of loop states. This is done by decomposing the representation on each edge as a product of fundamental representations, and then multiplying out the formulas for the invariants on nodes in terms of these products, and expanding.

The inner product of $\mathcal{H}^{k i n}$ is chosen so that the spin network states comprise an orthonormal basis

$$
<\Gamma \mid \Gamma^{\prime}>=\delta_{\Gamma \Gamma^{\prime}}
$$

This is of course natural, as such states are eigenstates of the operators that measure areas and volumes.

This completes the construction of the kinematical hilbert space $\mathcal{H}^{\text {kin }}$.

The next step is to construct a unitary realization of the diffeomorphism group, acting on $\mathcal{H}^{k i n}$. The rigorous theorems tell us this exists. At an intuitive level the construction proceeds as follows. Given a diffeomorphism $\phi \in \operatorname{Diff}(\Sigma)$ we represent it as a unitary operator as

$$
\hat{U}(\phi) \circ|\Gamma>=| \phi^{-1} \circ \Gamma>, \forall \phi \in \operatorname{Dif} f(\Sigma)
$$

It is trivial to check the unitarity. This follows from the fact that for any spin network $\Gamma$ and any diffeomorphism $\phi \in \operatorname{Diff}(\Sigma), \mid \Gamma>$ and $\mid \phi \circ \Gamma>$ are both normalizable states, with the same norm.

It is less trivial, but still true, to prove that there is no anomaly.

The next step is to change basis from the $<A \mid$ basis in which states are represented as functionals $\Psi(A)=<A \mid \Psi>$ to the spin network basis where they are functionals $\Psi(\Gamma)=<\Gamma \mid \Psi>$. Given that the $<\Gamma \mid$ comprise an orthogonal basis, this can be done rigorously. The functional transform

$$
\tilde{\Psi}(\Gamma)=\int d \mu(A) T[\Gamma, A] \Psi(A)
$$

is known precisely, the measure required is called the Ashtekar-Lewandowski measure. Heuristically this is analogous to the Fourier transform as $\langle A \mid \Gamma\rangle=T[\Gamma, A]$ gives a basis of states that the Hamiltonian acts simply on. Indeed, the key discovery that makes the quantization of general relativity possible is that the Hamiltonian and diffeomorphism constraints, which make up the hamiltonian, take any state $T[\Gamma, A]$ to a state of the same 
form, but with a different $\Gamma$. This is analogous to the fact that the Fourier transform is useful in ordinary quantum mechanics because the hamiltonian acts algebraically on momentum eigenstates.

One then defines a space of diffeomorphism invariant states

$$
\mathcal{H}^{\text {diffeo }} \subset \mathcal{H}^{\text {kin }}
$$

containing all states for which $\Psi[\phi \circ \Gamma]=\Psi[\Gamma]$, for all $\phi \in \operatorname{Diff}(\Sigma)$. It is easy to construct such states. For example, let $\mathcal{K}$ be any knot or graph invariant, then

$$
\Psi_{\mathcal{K}}[\Gamma]=\mathcal{K}[\Gamma]
$$

is in $\mathcal{H}^{\text {diffeo }}$. Similarly, let $\{\Gamma\}$ be the diffeomorphism equivalence class of the network $\Gamma$. Then the characteristic state defined by

$$
\Psi_{\{\Gamma\}}\left[\Gamma^{\prime}\right]=1 \text { if } \Gamma^{\prime} \in\{\Gamma\}
$$

and zero otherwise is in $\mathcal{H}^{\text {diffeo }}$. In fact, it can be shown that these provide an orthonormal basis of $\mathcal{H}^{\text {diffeo }}$.

Thus, the diffeomorphism invariant states perfectly combine the principles of duality and diffeomorphism invariance. Each state gives an amplitude to diffeomorphism classes of collections of Wilson loops. These states have physical meaning, given to them by the fact that they diagonalize diffeomorphism invariant observables that measure the geometry of the spatial slice. Examples of these which we mentioned above are the volume of the universe and the area of its boundary.

There is an unusual feature of the construction, worth commenting on. In a certain sense the kinematical Hilbert space, $\mathcal{H}^{\text {kin }}$ has too many states to represent a sensible physical theory. This is because any two spin network basis states are orthogonal, no matter by how little the graphs on which they are constructed differ. As a consequence it can be shown that there is no countable basis, so that the space is non-seperable.

This would be a disaster for physics. But we do not do physics in this space, we do physics in $\mathcal{H}^{\text {diffeo }}$, which is much smaller because we have identified all graphs that differ by a diffeomorphism. It can be shown that this space is seperable ${ }^{12}$.

To complete the theory one has to represent the dynamical evolution equations as operators on these diffeomorphism invariant states. This can be done in particular examples such as general relativity coupled to arbitrary matter fields in $3+1$ dimensions and supergravity. Exact expressions for the dynamical evolution of these states is known in both hamiltonian and path integral form. The action changes the graphs by local rules. A typical action is to act at a trivalent node, converting it to a triangle.

From the quantum Einstein equations we have exact closed form expressions for the amplitudes for these processes. This then gives rise to the picture of dynamics we sketched above in section 3.1.

We now turn to the methods by which the evolution amplitudes are constructed.

\footnotetext{
${ }^{12}$ if one mods out by piecewise smooth diffeomorphisms.
} 


\subsection{Dynamics of constrained topological field theories}

Observation IV means in details that all gravitational theories of interest, including general relativity and supergravity in 4 dimensions, can be described by an action, which is generically of the form ${ }^{13}$,

$$
S=S^{\text {topological }}+S^{\text {constraints }}+S^{\text {matter }}
$$

To describe the detailed form, its simplest first to fix the dimension to be four, in which case $G=S U(2)$.

The first term describes a topological theory called $B F$ theory. It depends on a 2 form $B^{i}$ and the field strength $F^{i}$ of a connection, $A^{i}$, all valued in a Lie algebra of $S U(2)$. Thus, $i=1,2,3$. The action is,

$$
S^{\text {topological }}=\int_{\mathcal{M}}\left(B^{i} \wedge F_{i}-\frac{\Lambda}{2} B^{i} \wedge B_{i}\right)
$$

The field equations which follow are

$$
\begin{gathered}
F^{i}=\Lambda B^{i} \\
\mathcal{D} \wedge B^{i}=0
\end{gathered}
$$

where $\mathcal{D}$ is the $S U(2)$ gauge covariant derivative.

The second term contains a quadratic function of $B^{i}$, which can be expressed as

$$
S^{\text {constraint }}=\int_{\mathcal{M}} \phi_{i j} B^{i} \wedge B^{j}
$$

where $\phi_{i j}$ is a symmetric, traceless matrix of scalar fields. Variation of the independent components of $\phi$ produces a quadratic equation in $B^{i}$ whose solution turns the theory into general relativity. These are

$$
B^{i} \wedge B^{j}=\frac{1}{3} \delta^{i j} B^{k} \wedge B_{k}
$$

The solutions to this are all of the following form: there exists a frame field $e_{a}^{I}$ for $I=$ $0, \ldots, 3=0, i$, such that the $B^{i}$ are the self-dual two forms of the metric associated to $e_{a}^{I}$. That is,

$$
B^{i}=e^{0} \wedge e^{i}+(\imath) \epsilon^{i j k} e_{j} \wedge e_{k}
$$

\footnotetext{
${ }^{13}$ This kind of formulation of general relativity was first discovered by Plebanksi[45] and later independently by $[46,47]$. The corresponding simplification of the Hamiltonian theory was independently discovered by Sen[1] and formalized by Ashtekar[2]. By now several different connections are used in loop quantum gravity. These include the self-dual part of the spacetime connection[1,2], and a real SU(2) connection introduced by Barbero[48] and exploited by Thiemann[49]. There are also alternate formulations that use both the left and right handed parts of the spacetime connection, [32, 50].
} 
where the $\imath$ is there for the Lorentzian case and not for the Euclidean case. Thus, the metric is not fundamental, instead the frame field appears when we solve the equations, (32), that constrain the degrees of freedom of the topological field theory.

The third term contains coupling to matter fields, such as spinors, scalars and YangMills fields. Interestingly enough, these can be written in terms of the fields involved in the other two terms[47].

It turns out that the same trick works in all dimensions, where $B^{i}$ is now a $d-2$ form. For $d=2+1$, the $B F$ theory is equivalent to general relativity[51]. For spatial dimension $d>3$ the extension has been given in [52]. It is also known how to express supergravity in $d=3+1$ [33] and $d=10+1$ [35] as constrained topological field theories.

Now we can return to the canonical quantization, and discover the structure we assumed above. Given a choice of time coordinate, $t$, which represents $\Sigma$ as constant time slicings ${ }^{14}$, we can find the canonical momenta to $A_{a}^{i}$. We see from the form of the action that the only time derivatives are in the first term,

$$
S^{\text {topological }}=\int d t \int_{\Sigma}\left(B^{i} \wedge \dot{A}_{i}+\ldots\right.
$$

Hence the canonical momenta to the gauge field are contained in conjugate electric fields, which can be expressed in terms of the pull back of the two forms of our theory to the spatial manifold $\Sigma$.

$$
E_{i}^{a}=\epsilon^{a b c} B_{b c i}
$$

where the $\epsilon^{a b c}$ is defined on the spatial manifold $\Sigma$, making $E^{i}$ a vector density on $\Sigma$. When the quadratic constraints from $S^{\text {constraints }}$ are solved, all the metric information is contained in the $B^{i \prime}$ s, and hence is represented by the electric field $E_{i}^{a}$ conjugate to the gauge field $A^{i}$. The gauge field $A_{a}^{i}$ will turn out to code components of the spacetime connection. This is a reversal of the older $A D M$ way of understanding the dynamics of the gravitational field, but it turns out to be deeper, and much more progress can be made with it.

We can easily see how the restriction from the topological $B F$ theory to general relativity by a quadratic equation works in the hamiltonian formulation. As the field equations of the $B F$ theory, $(29,30)$ are expressed as spacetime forms, they pull back to equations in the three surface $\Sigma$. These must hold the canonical theory. The covariant conservation of $B^{i}$ given by (30) pulls back, given (35), to Gauss's law

$$
\mathcal{G}^{i}=\mathcal{D}_{a} \tilde{E}^{a i}=0 .
$$

As in any Yang-Mills gauge theory, these are first class constraints that generate the ordinary gauge transformations. The field equation of the topological field theory (29) pulls back to

$$
\mathcal{F}_{a b}^{i}=F_{a b}^{i}+\Lambda \epsilon_{a b c} E^{c i}=0
$$

\footnotetext{
${ }^{14}$ However, among the gauge symmetries of the theory are diffeomorphisms of $\mathcal{M}$ that take any "spatial" slice representing $\Sigma$ to any other slice. If the theory implements the quantum version of the constraint that generates this gauge symmetry, it will not depend on the choice of slice used.
} 
These are constraints which tell us that the curvature is entirely determined by the $\tilde{E}_{i}^{a}$. Hence, the connection has no local independent degrees of freedom.

To give local dynamics to the connection we want to impose conditions on the fields, which will restrict the number of independent constraints. As mentioned above, to get general relativity in any dimension, it suffices to impose quadratic conditions on the $B^{i \text { 's. }}$ In fact, it is easy to see how this is realized in the Hamiltonian form of the theory, for the case of four spacetime dimensions.

The required quadratic constraints are equations of motion, hence they should be generated by the hamiltonian. This suggests that a hamiltonian at most cubic in fields should suffice. However in spacetime diffeomorphism invariant theories the hamiltonian must be a linear combination of constraints, so as to avoid any preferred time coordinates. On general grounds we expect there must be four more constraints, to generate the four dimensional diffeomorphisms.

These constraints should come from restricting the constraints (37) that give the topological field theory. The simplest way to do this turns out to lead to general relativity ${ }^{15}$. First, we can trace (37) with one power of the momenta, to find,

$$
D_{a}=\mathcal{F}_{a b}^{i} E_{i}^{b}=F_{a b}^{i} E_{i}^{b}=0
$$

It is easy to show that these generate the diffeomorphisms of $\Sigma$. The next simplest thing to do is to trace (37) with two powers of the momenta. This yields the desired cubic constraint,

$$
\mathcal{H}=\epsilon_{i j k} E^{a j} E^{b k} \mathcal{F}_{a b}^{i}=\epsilon_{i j k} E^{a j} E^{b k}\left[F_{a b}^{i}+\Lambda \epsilon_{a b c} E^{c i}\right]=0
$$

This is the Hamiltonian constraint. Remarkably, unlike older approaches, it is polynomial in the fields. This makes it possible to translate it into a quantum operator using nonperturbative methods ${ }^{16}$.

Can one stop here? One can if the constraints form a closed algebra. It turns out that the system of 7 constraints, consisting of (36),(38) and (39) do form a closed first class algebra. Hence it is consistent to impose only these four of the nine equations in (37). The result must be a theory with local degrees of freedom ${ }^{17}$ It is not hard to show that it is general relativity. It is also straightforward to show that for $d=4$ the constraints $(36,38,39)$ do follows from the action $(27)$.

\footnotetext{
${ }^{15}$ The same reasoning in the supersymmetric case leads to supergravity

${ }^{16}$ The form (39) holds for the Ashtekar-Sen variables, in which, for the Lorentzian case, $A_{a}^{i}$ is a complex variable. One can also work with the real Barbero variable, at a cost of an additional term added to the Hamiltonian constraint in the Lorentzian theory. However, it is still possible to convert the Hamiltonian constraint to a finite, well defined operator in this case.

${ }^{17}$ To count degrees of freedom, note that in the topological field theory Gauss's law (36) follows from (37), but this is not true of the restricted set $(38,39)$. Hence there is a net of two $(9-7)$ fewer equations, so there are two physical degrees of freedom. These are the polarizations of gravitational waves.
} 


\subsection{Horizons, black holes and boundaries}

Loop quantum gravity has led to a number of important results about black holes and cosmological horizons. In most of these results a horizon is modeled as a boundary of spacetime. Conditions are imposed at the boundary which capture the physical feature that at a horizon nulls rays neither diverge nor converge. As a result, only the degrees of freedom on the horizon and its exterior are quantized. This method certainly limits the kinds of questions that can be asked about black holes but, within this limitation, there is a complete description of the states associated with the black hole horizon.

These results are made possible by the relationship with topological field theory. The reason is easy to describe. Let us now assume that the spatial manifold $\Sigma$ has a boundary $\partial \Sigma=\mathcal{B}$ which is a closed, oriented two dimensional surface. For the classical and quantum dynamics to be well defined we must fix boundary conditions at $\mathcal{B}$ and we must also add a boundary term to the action of the theory. There is a very natural class of boundary conditions which follow from the relation to topological field theory. The reason is that the only derivatives of the action of general relativity are shared with the topological field theory. We can make use of natural relationships between topological field theories of different dimension, called the ladder of dimensions ${ }^{18}$. This works in any dimension, but there is an especially nice situation in $3+1$ dimension because the natural boundary theory associated with a $B F$ theory is a Chern-Simons theory on the three dimensional boundary of spacetime. To realize this we add a boundary term to (27), given by

$$
S^{\text {boundary }}=\frac{k}{4 \pi} \int_{\mathcal{B} \times R} Y_{C S}(A)
$$

where $Y_{C S}(A)$ is the Chern-Simons three form of the connection pulled back to the spacetime boundary. One can then show that the classical and quantum dynamics is well satisfied, so long as a boundary condition is satisfied on $\mathcal{B}$, which is[16]

$$
\left.B^{i}\right|_{\mathcal{B}}=\left.\frac{k}{2 \pi} F^{i}\right|_{\mathcal{B}}
$$

It turns out that this condition precisely characterizes black hole horizons, with the constant $k$ related to the surface gravity at the horizon[18]. it applies also to cosmological horizons[16] and to timelike boundary conditions in the case of a non-zero cosmological constant, of either sign[50]. In this case the consistency of the boundary condition (41) with the constraint (39) implies that,

$$
k=\frac{6 \pi}{G \hbar \Lambda} .
$$

Since Chern-Simons theory is precisely understood, this leads to a detailed understanding of the physics at these boundaries, including horizons[16]-[50].

\footnotetext{
${ }^{18}$ Whose relevance for quantum gravity was first emphasized by Louis Crane.
} 


\subsection{The path integral formulation: spin foam}

Path integral formulations of loop quantum gravity are known as spin foam models. There are several different constructions of them, and up to technical issues ${ }^{19}$, they yield the same class of theories. Each construction yields a spacetime history, or spin foam, $\mathcal{F}$ which evolves an initial spin network state $\mid \Gamma_{\text {in }}>$ into a final state $\left|\Gamma_{\text {out }}\right\rangle$. A spin foam history $\mathcal{F}$, is itself a combinatorial structure, whose boundary is the initial and final state

$$
\partial \mathcal{F}=\Gamma_{\text {out }}-\Gamma_{\text {in }}
$$

Here are several equivalent characterizations of a history, $\mathcal{F}$.

- A spin foam as a causal history. A causal spin foam is a succession of local moves $m_{i}$ that transform the initial state $\Gamma_{i n t}$ to the final state $\Gamma_{\text {out }}[53]-[57]$. The moves $m_{i}$ are considered the discrete analogues of the events of a continuous spacetime. They have a partial order, which give the history a discrete analogue of the causal structure of a Lorentzian spacetime. Consequently, each causal spin foam history is endowed with discrete analogues of structures such as light cones and horizons.

- A spin foam as a one dimension higher analogue of a Feynman diagram in which spin networks play the role normally played by incoming and outgoing particle states[58]. Propagators for edges are two dimensional spacetime surfaces, they meet along edges which are propagators for nodes. Each surface is labeled by a representation, as the edge it propagates. Similarly, each edge of the spin foam is labeled by an invariant corresponding to the node of the spin network it propagates. Interactions occur at vertices in the foam, where several edges meet. Any cross section of such a spin foam is a spin network. The interactions are events where the spin network changes by a local move.

- Spin foams as Feynman diagrams of a matrix model. The analogy to Feynman diagrams is made precise by showing that there for every spin foam model there is a matrix model (more precisely a dynamical system on a group manifold) such that the spin foams are the Feynman diagrams of that matrix model[59]. Each foam is then a Feynman diagram, and like in ordinary quantum field theory, there are sums over intermediate states on internal propagators. In the spin foam case these are sums over representation labels on two surfaces and sums over invariant labels on edges. But unlike ordinary $Q F T$, in a large class of physically relevant models, the sums over intermediate states are finite.

- A spin foam as a triangulation of spacetime For simplicity, consider the case where the initial and final spin network are four valent graphs. They then are each dual to a three dimensional triangulation ${ }^{20}$ in which each node is dual to tetrahedra,

\footnotetext{
${ }^{19}$ For the interested reader these are described below.

${ }^{20}$ Technically, a pseudo-manifold,
} 
whose four faces are dual to the four edges incident on that node[60, 61]. Each face of the dual triangulation is now labeled by a representation, while each tetrahedron is labeled by an invariant. There are then spin foams constructed from a four dimensional triangulation, whose boundary is the three triangulation dual to the initial and final spin network. Each four simplex is dual to an event in the previous characterizations of a spin foam.

Following the rules of quantum theory, the dynamics of the theory is specified when we have assigned an amplitude to each history. The amplitude of each foam $\mathcal{A}[\mathcal{F}]$ is given by a product

$$
\mathcal{A}[\mathcal{F}]=\mathcal{N}^{-1} \prod_{\text {events }} \mathcal{A}[\text { event }]
$$

of factors for each event (or vertex or four-simplex), where each amplitude is a function of the labels of surfaces and edges incident at that event,. $\mathcal{N}$ is a normalization factor which depends on the labels on lower dimensional structures. Several models have been studied as candidates for quantum general relativity. The best studied is the Barrett-Crane model, which is derived by following a strategy which is naturally indicated by the realization that general relativity is a constrained topological field theory. The first step is to define a spin foam model which exactly realizes the quantization of the $B F$ theory given in (28).

The second step is to implement the quadratic constraint, (32), on the sums over labels in the path integral representation of the topological field theory. It turns out there is a very beautiful and natural way to do this. The result is that the path integral measure for quantum general relativity is exactly the same as the measure of the corresponding topological field theory. The only difference is that in quantum general relativity the sum over labels is restricted to a subset of representations and invariants. This restriction implements the quadratic constraint and, by destroying the topological invariance, leads to a theory with local degrees of freedom. This simple construction is known to work for quantum general relativity in all dimensions, for both Lorentzian and Euclidean theories[52].

Following the conventional rules of quantum theory, amplitudes of physical interest are to be constructed from summing the corresponding amplitudes over all spin foam histories. Thus, we have

$$
<\Gamma_{\text {out }} \mid \Gamma_{\text {in }}>=\sum_{\mathcal{F} \text { s.t. } \partial \mathcal{F}=\Gamma_{\text {out }}-\Gamma_{\text {in }}} \mathcal{A}[\mathcal{F}]
$$

A spin foam model then provides a precise realization of the sum over spacetime histories, conjectured and described formally in early work by Hawking, Hartle and others. Given such a model, one can use it to construct different amplitudes of interest including projection operators onto the space of physical states, annihilated by all the constraints. One can construct as well physical evolution amplitudes, such as the amplitude to evolve from the initial state to the final state through histories with a fixed spacetime volume. 


\section{The main results of loop quantum gravity}

We can now turn to listing some of the main results which have been obtained concerning loop quantum gravity and spin foam models.

\subsection{The fundamental results of the canonical theory}

The first set of results concern the construction of a Hilbert space of states of a gauge field valued in a Lie algebra or superalgebra $\mathcal{A}$, invariant under local gauge transformations and diffeomorphisms of $\Sigma$.

The setting for the results that follow is a bare differentiable manifold $\Sigma$, with no metric structure, on which is defined the phase space of a gauge theory, with gauge group $G$. The only non-dynamical structure that is fixed is a three manifold $\Sigma$, with a given topology and differential structure. There are no fixed classical fields such as metrics, connections or matter fields on $\Sigma$. The only exception is in modeling the quantization of spacetime regions with boundary, as in the asymptotically flat or $A d S$ context, or in the presence of a black hole or cosmological horizon. In these cases fields may be fixed on the boundary $\partial \Sigma$ to represent physical conditions held fixed there. The dynamics is formulated in terms of a diffeomorphism invariant action which is a functional only of that gauge field and its derivatives.

1. The states of the theory are known precisely[69, 70, 71, 72, 7, 6]. The kinematical Hilbert space $\mathcal{H}^{k i n}$ has been rigorously constructed and has all the properties described in the last section. The Hilbert space $\mathcal{H}^{\text {diffeo }}$ of spatially diffeomorphism invariant and gauge invariant states of a gauge field on a manifold $\Sigma$, has an orthonormal basis, $\mid\{\Gamma\}>$ whose elements are in one to one correspondence with the diffeomorphism equivalence classes of embeddings of spin networks[62], into $\Sigma$ [63]. The inner product is given by

$$
<\{\Gamma\} \mid\left\{\Gamma^{\prime}\right\}>=\delta_{\{\Gamma\}\left\{\Gamma^{\prime}\right\}} .
$$

Here $\{\Gamma\}$ refers to the equivalence class of graphs under piecewise smooth diffeomorphisms of $\Sigma$. In the case of pure general relativity in $3+1$ dimensions, with vanishing cosmological constant, the gauge group is $S U(2)$. In this case the labels on the edges are given by ordinary $S U(2)$ spins. For details see $[62,63]$.

2. Certain spatially diffeomorphism invariant observables have been constructed. After a suitable regularization procedure these turn out to be represented by finite operators on $\mathcal{H}^{\text {diffeo }}$, the space of spin network states[10, 64, 65]. In the case of general relativity and supergravity, these include the volume of the universe, the area of the boundary of the universe, or of any surface defined by the values of matter fields. These operators all preserve the diffeomorphism invariance of the states[68].

Other operators also have been constructed, for example an operator that measures angles in the quantum geometry[73] and the lengths of curves in $\Sigma[74]$. 
3. The area, volume and length operators have discrete, finite spectra, valued in terms of the Planck length $[64,65,66,74]$. There is hence a smallest possible volume, a smallest possible area, and a smallest possible length, each of Planck scale. The spectra have been computed in $[66,67]$.

4. The area and volume operators can be promoted to genuine physical observables, by gauge fixing the time gauge so that at least locally time is measured by a physical field $[75,68]$. The discrete spectra remain for such physical observables, hence the spectra of area and volume constitute genuine physical predictions of the quantum theory of gravity.

5. Among the operators that have been constructed and found to be finite on $\mathcal{H}^{\text {diffeo }}$ is the Hamiltonian constraint (or, as it is often called, the Wheeler de Witt equation[76]-[78].) Not only can the Wheeler deWitt equation be precisely defined, it can be solved exactly. Several infinite sets of solutions have been constructed, as certain superpositions of the spin network basis states, for all values of the cosmological constant[70, 49]. These are exact, physical states of quantum general relativity.

6 If one fixes a physical time coordinate, in terms of the values of some physical fields, one can also define the Hamiltonian for evolution in that physical time coordinate[75] and it is also given by a finite operator on a suitable extension of $\mathcal{H}^{\text {diffeo }}$ including matter fields.

8. There is a rigorous formulation of the Hamiltonian quantization of general relativity in which all the preceding results are reproduced as theorems[72, 49, 7]. In the context of this rigorous approach, there is a fundamental uniqueness theorem[31], which essentially says the following ${ }^{21}$ :

\footnotetext{
${ }^{21}$ A more precise statement of the theorem follows[79].
}

Let $A(e)$ be the holonomy of a classical connection along a piecewise analytic path $e$, let $E_{f}(S)=\int_{S} \operatorname{Tr}(f *$ $E$ ) be the electric flux of the classical two-form $* E$ through the piecewise analytic surface $S$ where $f$ s a Lie algebra valued smooth scalar field. Let $D$ be the group of piecewise analytic spatial diffeomorphisms of the hypersurface and $G$ the group of local gauge transformations. Let $W$ be the Weyl algebra generated by $\left[A(e), A\left(e^{\prime}\right)\right]=0$ and $W_{f}(S) A(e) W_{f}(S)^{-1}=\exp \left(i \hbar \mathcal{L}_{\chi_{E_{f}(S)}}\right) \cdot A(e)$.

where we have, $A(e)^{*}=A\left(e^{-1}\right)^{T}$ (T=transposition of matrices) $W_{f}(S)^{*}=W_{-f}(S)$

Finally consider group of automorphisms of $W$ labeled by the semidirect product $G \times D$ given by $\alpha_{g}(A(e))=g(b(e)) A(e) g(f(e))^{-1}(g \in G ; b(e), f(e)$ beginning and end point of $e) \alpha_{d}(A(e))=A(d(e))$ and similar for $W_{f}(S)$.

We are looking for representations $\pi$ of the *algebra $W$ on a Hilbert space $H$ which

1. contains a cyclic vector $\Omega$, i.e the states $\pi(a) \Omega$ span $H$ as we let $a$ vary through $W$.

2. implements $G \mid x D$ unitarily by $U(g, d) \pi(a) \Omega=\pi\left(\alpha_{g, d}(a)\right) \Omega$ for all $a \in W$

3. is weakly cont. wrt $t \rightarrow \pi\left(W_{t f}(S)\right)$ for all $f, S$ for real parameter $t$, and that $\Omega$ is in the common dense domain of all the $\pi\left(E_{f}(S)\right.$. That means that matrix elements of $\pi\left(W_{t f}(S)\right.$ become matrix elements of the unit operator ast $\rightarrow 0$ which by Stone's theorem means that the flux operators $\pi\left(E_{f}(S)\right)$ exist. This technical assumption is necessary also in Stone von Neumann's theorem without which the uniqueness of the Schroedinger rep of QM is wrong.

A different way of saying this is that $(\pi, \Omega, H)$ are the GNS data for a state (pos. lin. functional) $\omega$ on $W$ satisfying the invariance condition $\omega \circ \alpha_{g, d}=\omega$ and the continuity assumption. 
Consider an approach to a quantum gauge theory on a $d \geq 2$ dimensional manifold without metric. Assume that the Wilson loop operator and area (or generally electric flux) operator are well defined on a kinematical hilbert space, $\mathcal{H}^{\text {kin }}$. Assume also that $\mathcal{H}^{\text {kin }}$ carries a unitary anomaly free representation of Dif $f(\Sigma)$ so that the space $\mathcal{H}^{\text {diff }}$ of diffeomorphism invariant states may be constructed (formally as a subspace of a dual space of $\mathcal{H}^{\text {kin }}$. Then the Hilbert space of the theory is isomorphic to that just described.

\subsection{Results on path integrals and spin foams}

9. The dynamics of the spin network states can be expressed in a path integral formalism, called spin foams[13]-[15],[53]-[61] $]^{22}$. The histories by which spin network states evolve to other spin network states, called spin foam histories, are explicitly known. A spin foam history is a labeled combinatorial structures, which can be described as a branched labeled two complex. Spin foam models have been derived in several different ways, and the results agree as to the general form of a spin foam amplitude. These include: 1) by exponentiation of the Hamiltonian constraint, 2) directly from a discrete approximation to the classical spacetime theory, 3) by constraining the summations in a finite state sum formulation of a four dimensional topological invariant, 4) from a matrix model on the space of fields over the group, 5) by postulating spacetime events are local moves in spin networks.

10. Evolution amplitudes corresponding to the quantization of the Einstein equations in $3+1$ dimensions, are known precisely[61, 15] for vanishing and non-vanishing values of the cosmological constant, and for both the Euclidean and Lorentzian theories.

11. The sum over spin foams has two parts, a sum over graphs representing histories of spin networks, and, on each, a sum over the labels. The sums over labels are known from both analytic and numerical results to be convergent[80, 81] for some spin foam models, including some corresponding to the quantization of the Einstein equations in $2+1$ and $3+1$ dimensions.

12. For some spin foam model in $2+1$ dimensions, it has been shown that the sum over spin foam histories is Borel summable[82].

13. The physical inner product, which is the inner product on solutions to all the constraints, has an exact expression, given in terms of a summation over spin foam amplitudes[58].

14. The spin foam models have been extended to include gauge and spinor degrees of freedom.

Then the THEOREM is: There is only one solution, the AILRS Hilbert space. Moreover, the representation $\pi_{A I L R S}$ is irreducible (that is, not only one vector is cyclic but every vector is cyclic) which excludes the existence of spurious superselection sectors.

${ }^{22}$ For the most recent review see [15]. 
15. Spin foam models with matter have been extensively studied in $2+1$ dimensions[83]. It has been shown that the limit $G_{N} \rightarrow 0$ reproduces the Feynman diagram expansion of the matter quantum field theory on $2+1$ dimensional Minkowski spacetime. For $G>0$ the matter quantum field theory on $2+1$ dimensional $\kappa$-Minkowski spacetime is reproduced. This shows that at least in $2+1$ dimensions the scattering of matter coupled to quantum gravity is described by a version of deformed special relativity. This is discussed more in sections 4.5 and 5 .

16. Spin foam models appropriate for Lorentzian quantum gravity, called causal spin foams, have quantum analogues of all the basic features of general relativistic spacetimes $^{23}$. These include dynamically generated causal structure, light cones and a discrete analogue of multifingered time, which is the freedom to slice the spacetime many different ways into sequences of spatial slices[53]. The spatial slices are spin networks, which are quantum analogues of spatial geometries ${ }^{24}$.

\subsection{Matter and unification}

17. Coupling to all the standard forms of matter fields are understood, including gauge fields, spinors, scalars and higher $p$-form gauge fields ${ }^{25}[6,7]$. It is known how to extend the definition of the spatially diffeomorphism invariant states to include all the standard kinds of matter fields, and the corresponding terms for the Hamiltonian constraint are known in closed form, and are finite on the space of diffeomorphism invariant states. These states are also invariant under ordinary Yang-Mills and $p$-form gauge transformations ${ }^{26}$. Inclusion of matter fields does not affect the finiteness and discreteness of the area and volume observables.

\subsection{Results on black holes and horizons}

18. Several kinds of boundaries may be incorporated in the theory including timelike boundaries, in the presence of both positive and negative cosmological constant, and null boundaries such as black hole and cosmological horizons[16]-[18, 32, 50]. In all these cases the boundary states and observables are understood in terms of structures derived from

\footnotetext{
${ }^{23}$ For more details on these models and the resulting physical picture, see[54].

${ }^{24}$ It should be mentioned also that there are very impressive results relevant to the spin foam program from a research program called causal dynamical triangulations. This work shows that Lorentzian and Euclidean path integrals for quantum gravity fall into different universality classes, with the Lorentzian path integrals being more convergent[39]-[43]. For the first time there are results that suggest that a dynamical $3+1$ spacetime may emerge from critical behavior of a path integral over combinatorially defined spacetimes[43].

${ }^{25}$ For supergravity, see result 31 , below.

${ }^{26}$ To my knowledge whether loop quantum gravity suffers from the fermion doubling problem is an open question.
} 
Chern-Simons theory. The boundary theory provides a detailed microscopic description of the physics of horizons and other boundaries.

19. The horizon entropy (47) is completely explained in terms of the statistical mechanics of the state spaces associated with the degrees of freedom on the horizon. This has been found to work for a large class of black holes, including Schwarzschild black holes[17, 18]. LQG also gives the correct results for dilatonic black holes[94].

The boundary Hilbert spaces decompose into eigenspaces, one for each eigenvalue of the operator that measures the area of the boundary[16]. For each area eigenvalue, the Hilbert space is finite dimensional. The entropy may be computed and it agrees precisely with the Bekenstein-Hawking semiclassical result,

$$
S=\frac{A[S]}{4 \hbar G_{\text {Newton }}}
$$

The calculated entropy is proportional to a parameter, which is called the Imirzi parameter. This can be understood either as a free parameter that labels a one parameter family of spin network representations, or as the (finite) ratio of the bare to renormalized Newton's constant.

The Imirzi parameter can be calculated by computing the entropy statistically. To do this one has to make an assumption about which microstates of the horizon should be counted as corresponding to the macrostate which is the Schwarzchild black hole. Two different answers, both of order one have been gotten depending on which assumption is made[86, 87]

The Imirzi parameter can also be fixed by an argument invented by Dreyer, involving quasi normal modes of black holes[84]. The value gotten by this method matches one of the values gotten by calculating the ratio of microstates to macrostates explicitly.

Dreyer's argument depends on a remarkable and precise coincidence between an asymptotic value of the quasi normal mode frequency and a number which appears in the loop quantum gravity description of horizons. The value of the asymptotic quasi normal mode frequency was at first known only numerically, but it has been very recently derived analytically by Motl[85]. Once Dreyer's argument fixes the Imirzi parameter, the Bekenstein-Hawking relation (47) is predicted exactly for black hole and cosmological horizons.

20. Corrections to the Bekenstein entropy have been calculated and found to be logarithmic[95].

21To study the problem of the fate of the singularity models of the interior of a spherically symmetric black hole horizon have been studied $[88,89$, 93]. These are $1+1$ dimensional field theories. It is found that the black hole singularities are eliminated[88, 89]. From this one can construct a plausible answer to the problem of loss of information in black hole evaporation[93].

22. Suitable approximate calculations reproduce the Hawking radiation. They further predict a discrete fine structure in the Hawking spectrum[96, 97]. At the same time, the 
spectrum fills in and becomes continuous in the limit of infinite black hole mass. This fine structure stands as another definitive physical prediction of the theory.

Thus, to summarize, loop quantum gravity leads to a detailed microscopic picture of the quantum geometry of a black hole or cosmological horizon[18]. This picture reproduces completely and explains the results on the thermodynamic and quantum properties of horizons from the work of Bekenstein[98], Hawking[99] and Unruh[100]. This picture is completely general and applies to all black hole and cosmological horizons.

\subsection{Results on the low energy behavior}

23. A large class of putative ground states can be constructed in $\mathcal{H}^{k i n}$ which have course grained descriptions which reproduce the geometry of flat space, or any slowly varying metric[101]-[106]. Linearizing the quantum theory around these states yields linearized quantum gravity, for gravitons with wavelength long compared to the Planck length[104].

24. Excitations of matter fields on these states reproduce a cut off version of the matter quantum field theories, but with a physical, Planck scale cutoff. As a result of the discreteness of area and volume, the ultraviolet divergences of ordinary QFT are not present[7].

25. It is understood rigorously how to construct coherent states in $\mathcal{H}^{\text {kin }}$ which are peaked around classical configurations[106].

26. Formulations of the renormalization group for spin foam models have been given in $[107,108]$. As a byproduct of this work it is shown that while the Wilsonian renormalization group is not a group, it does have a natural algebraic setting, as a Hopf algebra.

27. For the case of non-vanishing cosmological constant, of either sign, there is an exact physical state, called the Kodama state, which is an exact solution to all of the quantum constraint equations, whose semiclassical limit exists[109]. ${ }^{27}$ That limit describes deSitter or anti-deSitter spacetime. Solutions obtained by perturbing around this state, in both gravitational[11] and matter fields[110], reproduce, at long wavelength, quantum field theory in curved spacetime and the quantum theory of long wave length, free gravitational waves on deSitter or anti-deSitter spacetime ${ }^{28}$.

It is not yet known whether or not the Kodama state can be understood in a rigorous setting, as the state is not measurable in the Ashtekar-Lewandowski measure which is an essential element of the rigorous approach to the canonical quantization. At the same tine, the integrals involved are understood rigorously in terms of conformal field theory. It is thus not known if the state is normalizable in the physical inner product of the exact theory. One can ask whether the projection into the Hilbert space of linearized gravitons

\footnotetext{
${ }^{27} \mathrm{~A}$ related state in the context of Yang-Mills theory was previously discussed by Jackiw[114]. Some properties of Jackiw's state were discussed by Witten[115]. More about this in a FAQ.

${ }^{28}$ For a possible $\Lambda=0$ analogue of the Kodama state, see [113].
} 
on deSitter spacetime gotten by truncating the Kodama state to quadratic order is normalizable or not. The answer is it is not in the Lorentzian case, and it is delta functional normalizable in the Euclidean case[116].

28. The inverse cosmological constant turns out to be quantized in integral units, so that

$$
k=6 \pi / G \Lambda
$$

is an integer[16]. This is related to a basic result, which is that in $3+1$ dimensions, a nonzero cosmological constant implies a quantum deformation of the gauge group whose representations label the edges of the spin networks, where the level which parameterizes the quantum deformation is given for the Euclidean case by eq. $(48)^{29}$.

29. The thermal nature of quantum field theory in a deSitter spacetime is explained in terms of a periodicity in the configuration space of the exact quantum theory of general relativity[110, 11].

30. In both flat space and around deSitter spacetime, one may extend the calculations that reproduce quantum theory for long wavelength gravitons and matter fields to higher energies. These calculations reveal the presence of corrections to the energy-momentum relations of the form of

$$
E^{2}=p^{2}+M^{2}+\alpha l_{P l} E^{3}+\beta l_{P l}^{2} E^{4}+\ldots
$$

Given a candidate for the ground state, the parameters $\alpha$ and $\beta$ are computable constants, that depend on the ground state wavefunctional[22, 23, 11]. Thus, given a choice for the ground state the theory yields predictions for modifications of the energy momentum relations.

As discussed above, in the $2+1$ dimensional theory, modifications of the energy momentum relations (49) are present. They are understood as indicating, not a breaking of Poincare invariance, but a deformation of it[117]. It is conjectured[11, 118], but not shown, that the same will be true of quantum gravity in $3+1$ dimensions. This will be discussed further in the next section.

To summarize, the situation with regard to the low energy limit is very much like that of condensed matter systems. It is possible to invent and study candidates for the ground state, which have reasonable physical properties and reproduce the geometry of flat or deSitter spacetime. By studying excitations of these states one reproduces conventional quantum field theory, as well corrections to it which may be compared with experiment.

There is not so far a first principles calculation that provides an exact or unique form of the ground state wavefunction. This is also the case in most condensed matter physics examples. It remains an open question whether one can derive first principles or model independent physical predictions for the energy momentum relations (49) from the theory. This will be discussed further below.

\footnotetext{
${ }^{29}$ and for the Lorentzian case by $\imath k$.
} 


\subsection{Results concerning cosmology}

31. An approach to quantum cosmology has been developed from applying the methods of loop quantum gravity to the spatially homogeneous case[20]. This is called loop quantum cosmology, (LQC). There is a physical (but so far not rigorous) argument that the states studied in this reduced approach correspond to spatially homogeneous states in the Hilbert spaces, $\mathcal{H}^{\text {diff }}$ of the full theory. The space of states of the homogeneous theory is motivated by the fact that normalizabie states of the full theory, based on Wilson loops, reduce to normalizable states of the reduced theory.

This means that the Hilbert space of LQC is not (for the simplest models, that just depend on the scale factor, a) $\mathcal{L}_{2}\left(R^{+}\right)$as is the case in most semiclassical approaches to quantum cosmology. Instead, it is a Bohr quantization, in which $A$ is not a well defined operator, but $e^{A}$ is. As a result, the results differ from those of older approaches to quantum cosmological models.

32. The evolution of states of this reduced theory has been studied in detail and it has been found generically that the usual FRW cosmology is reproduced when the universe is very large in Planck units. At the same time, the cosmological singularities are removed, and replaced by bounces where the universe re-expands (or pre-contracts).

The replacement of cosmological singularities by bounces has been confirmed in several different models of this type, that make different assumptions as to symmetries and dynamics, and hence appears robust[40,89].

It is not yet known rigorously whether the singularities are eliminated in the full theory. But it has been shown that some of the properties of the density operator in the LQC model are carried over into the full theory, including the fact that the density operator is finite on states of the theory that have zero volume[90]

33. When couplings to a scalar field are included, there is a natural mechanism which generates Planck scale inflation as well as a graceful exit from it[20]. Using this formulation of quantum cosmology it has recently it has been argued that loop quantum gravity effects lead naturally to a version of chaotic inflation which may also explain the lack of power on long wave lengths in the $C M B$ spectrum[21].

34 The transplanckian corrections to the spectrum of fluctuations have been computed in one such model[91,92]. At least in this one model, order $l_{P l} H$ corrections vanish, while there are explicitly computer corrections at order $\left(l_{P l} H\right)^{2}$, where $H$ is the Hubble constant during inflation.

35. Another approach to inflation within loop quantum gravity is given in [119]. Exact homogeneous quantum states can be constructed for general relativity coupled to a scalar field with an arbitrary potential. This may be used to construct exact quantum states corresponding to inflating universes, in the homogeneous approximation.

36. It has been shown that loop quantum gravity effects eliminate the chaotic behavior 
of Bianchi IX models near singularities[120].

37 A mechanism analogous to the Pecci-Quinn mechanism has been proposed for relaxation of the cosmological constant[121].

\subsection{Results concerning supergravity and other dimensions}

38. Many of these results extend to quantum supergravity for $N=1$ and several have been studied also for $N=2[33,34,32]$.

39. The same methods can also be used to solve quantum gravity in $2+1$ dimensions[51] and in some $1+1$ dimensional reductions of the theory[131]. They also work to solve a large class of topological field theories[132, 133], giving results equivalent to those achieved by other methods. Further, loop methods applied to lattice gauge theories yields results equivalent to those achieved by other methods[134].

40. Spin foam models are known in closed form for quantum general relativity in an arbitrary dimension $d>4$ [52].

41 There is a class of diffeomorphism invariant theories in 6 and 7 dimensions whose degrees of freedom are just forms[135]. These have been conjectured to describe a diffeomorphism invariant theory of interest to string theorists called Topological $\mathcal{M}$ theory[136]. This has been quantized using LQG methods[137].

\subsection{Other extensions of the theory}

42. The mathematical language of spin networks and spin foams can be used to construct a very large class of background independent quantum theories of gravity. These may be called generalized loop quantum gravity models. The states of such a quantum theory of gravity are given by abstract spin networks ${ }^{30}$.

The graphs on which the spin networks are based are defined combinatorially, so that the need to specify the topology and dimension of the spatial manifold is eliminated[53, 56]. In such a theory the dimension and topology are dynamical, and different states may exist whose coarse grained descriptions reveal manifolds of different dimensions and topology.

The histories of the theory are given by spin foams labeled by the same representations. The dynamics of the theory is specified by evolution amplitudes assigned to the nodes of the spin foams (or equivalently to local moves by which the spin networks evolve).

Such generalized loop quantum gravity models have been proposed to serve as background independent formulations of string and $\mathcal{M}$ theory[138, 56]. They also offer new

\footnotetext{
${ }^{30} \mathrm{An}$ abstract spin network is a combinatorial graph whose edges are labeled by representations of $\mathcal{A}$ and whose nodes are represented by invariants of $\mathcal{A}$. associated with the representation theory of a given Hopf algebra or superalgebra, $\mathcal{A}$
} 
possibilities for the unification of physics, because the topology of spacetime is not assumed a priori, and hence must be emerge dynamically in the low energy limit. There is then the possibility of new physics coming from obstructions to recovering trivial topology at low energies. It has been proposed that these may account for the existence of matter degrees of freedom[139, 140], and perhaps even quantum theory itself[142].

\subsection{Comments on the results}

On the basis of these results, it can be claimed that loop quantum gravity is both a consistent quantization of general relativity and a physically plausible candidate for the quantum theory of gravity.

The failure of quantum general relativity in perturbation theory is explained by the fact that there are, in this quantization of general relativity, no degrees of freedom that correspond to gravitons or other perturbative quanta with wavelength shorter than the Planck scale. The ultraviolet divergences are eliminated because a correct quantization, that exactly realizes spatial diffeomorphism invariance, turns out to impose an ultraviolet cutoff on the physical spectrum of the theory. The assumptions, made by other approaches, that spacetime is smooth and lorentz invariant at arbitrarily short scales, are not used in the quantization procedure, and in fact turns out to be contradicted by the results.

\section{The near term experimental situation}

The most important development of the last few years in quantum gravity is the realization that it is now possible to probe Planck scale physics experimentally[143]-[161]. These experiments look for modifications in the energy momentum relations of the form of eq. (49).

However it is crucial to mention that to measure $\alpha$ and $\beta$ of eq. (49) one has to specify how lorentz invariance is treated in the theory. There are two very different possibilities which must be distinguished.

- Scenario A) The relativity of inertial frames is broken and there exists a preferred frame. In this case the analysis has to be done in that preferred frame. The most likely assumption is that the preferred frame coincides with the rest frame of the cosmic microwave background. In such theories energy and momentum conservation are assumed to remain linear.

- Scenario B) The relativity of inertial frames is preserved, but the lorentz transformations are realized non-linearly when acting on the energy and momentum eigenstates of the theory. Such theories are called modified special relativity or doubly 
special relativity[146, 149, 150,148]. Examples are given by some forms of noncommutative geometry, for example, $\kappa$-Minkowski spacetime[147]. In all such theories energy and momentum conservation become non-linear which, of course, effects the analysis of the experiments.

Among the experiments which either already give sufficient sensitivity to measure $\alpha$ and $\beta$, or are expected to by 2010 , are,

1. There are apparent violations of the GZK bound observed in ultra high energy cosmic rays (UHECR) detected by the AGASA experiment[144]. The experimental situation is inconsistent, but the new AUGER cosmic ray detector, which is now operational, is expected to resolve the situation over the next several years. If there is a violation of the GZK bound, a possible explanation is Planck scale physics coming from (49) [145].

In Scenario A) violations of the GZK bounds can be explained by either $E^{3}$ or $E^{4}$ terms in the proton energy-momentum relation. However, in Scenario B) it is less natural to explain a violation of the GZK bounds by means of a Planck scale modification of the energy-momentum relations, but there are proposals for forms of such theories that do achieve this.

2. A similar anomaly is possibly indicated in Tev photons coming from blazers[153]. Similar remarks apply as to the explanatory power of Scenarios A) and B) in the event that the anomaly exists.

3. A consequence of (49) can be an energy dependent speed of light. This effect can be looked for in timing data of gamma ray busts. Present data bounds $\alpha<\approx 10^{4}$ [155] and data expected from the GLAST experiment is expected to be sensitive to $\alpha$ of order one in 2006 [154]. Note that this applies to both Scenarios A) and B).

4. Present observations of synchrotron radiation in the Crab nebula, together with reasonable astrophysical assumptions, put very strong (of order $10^{-9}$ ) bounds on $\alpha$ for photons and electrons, in the case of Scenario A only[156].

5. Present data from precision nuclear and atomic physics experiments puts very tight bounds on $\alpha$ for photons, electrons and hadrons, again in Scenario A), only[157].

6. Present data from the absence of vacuum Cherenkov effects puts interesting bounds on $\alpha$ in the case of Scenario A) [151].

7. Observations of bifringence effects in polarized light from distant galaxies puts tight bounds on a possible helicity dependent $\alpha$ [158].

8. Observations of phase coherence in stellar and galactic interferometry is expected, given certain assumptions ${ }^{31}$, to put order one bounds on $\alpha$ in the near future [159].

\footnotetext{
${ }^{31}$ See [160] for discussion of them.
} 
9. Certain hypotheses about the Planck scale lead to the prediction of noise in gravitational wave detectors that may be observable at LIGO and VIRGO[161].

10. Under some cosmological scenarios, modifications of the form of (49) lead to distortions of the CMB spectrum that may be observable in near future observations[162].

11. There is a possibility that observations of very high energy neutrinos from cosmological distances, in experiments such as ICEBERG may make it possible to test for both violations of special relativity and loss of quantum coherence[122].

We may summarize this situation by saying that a theory of quantum gravity that leads to Scenario A) and predicts an energy momentum relation (49) with $\alpha$ order unity is plausibly already ruled out. This is shocking, as it was commonly said just a few years ago that it would be impossible to test any physical hypotheses concerning the Planck scale.

We can also mention three other kinds of experiments that by 2010 will have relevance for the problem of quantum gravity

1. Evidence for or against supersymmetry may be detected at the Tev scale at the LHC.

2. The equation of state of the dark energy will be measured in near future experiments. Some proposals for dark energy[164] are based on modifications of energy momentum relations of the form of (49).

3. There are observations that appear to indicate that the fine structure constant is time dependent[163]. These will be confirmed or go away. If the claim is substantiated this offers a big challenge to the effective field theory understanding of low energy physics.

The combination of all these experimental possibilities signals that the long period when fundamental physics developed independently of experiment is soon coming to a close.

\subsection{What does loop quantum gravity predict for the experiments?}

It is first important to observe that there is no reason that the low energy limit of quantum gravity must be Poincare invariant. Poincare invariance is not a symmetry of general relativity, it is only a global symmetry of a particular solution of the classical theory. Global symmetries including Poincare and Lorentz invariance are not symmetries of the fundamental theory of gravity, neither classically nor quantum mechanically. Whether these symmetries are fully realized in the ground state has to be determined by calculation.

Indeed, several recent calculations, done with different methods[22, 23, 11], yield predictions for modifications to the energy momentum relations for elementary particles of 
the form of equation (49). Predictions have been found for the leading coefficients $\alpha$, which generally depend on spin and helicity[22]-[11].

One issue here is that different calculations make different assumptions about the ground state. In some the ground state is not Lorentz invariant, hence there is no surprise if the perturbations around them have non-lorentz invariant spectra. However, modified dispersion relations may also be seen by studying low energy excitations of a putative around state that does not single out a preferred frame[11]. The question is then: can we determine the ground state precisely enough to discover whether the theory makes unambiguous predictions for the parameters in the energy-momentum relations (49)? Of particular importance is to determine which scenario is realized, A) or B) ?.

There is a straightforward argument leading to an expectation that Scenario B) is realized. This is that, even though there is no global lorentz invariance in classical general relativity, the existence of effects due to a preferred frame are ruled out by the condition of invariance under the action of the hamiltonian constraint. This is because the hamiltonian constraint can generate changes in slicing that in any finite region are indistinguishable from lorentz boosts.

Now some of the key results of loop quantum gravity tell us that the hamiltonian constraint can be defined and solved exactly, and that no anomalies are introduced into the constraint algebra by the quantization. This implies that any quantum state that is both an exact solution to the hamiltonian constraint and has a semiclassical limit will in that limit describe physics which is to leading order invariant under the action of the classical hamiltonian constraint. This implies the absence of a preferred frame of reference in the classical limit of an exact solution to the hamiltonian constraint.

The only exception to this could be if there were a vector or tensor field in the theory that could pick up a vacuum expectation value in the ground state, essentially breaking Lorentz invariance spontaneously. The field in question must be able to get a vacuum expectation value without breaking any gauge invariance. Thus, it cannot be the metric field itself, for if it had an expectation value it would break also diffeomorphism invariance. This cannot happen as diffeomorphism invariance is a gauge symmetry. Nor can it be an ordinary gauge field. Thus,spontaneous breaking of Lorentz invariance cannot be generic, it can only be due to a special matter dynamics being imposed on the theory.

So this appears to rule out Scenario A), in all but a few artificial cases.

This reasoning is supported by many results on $2+1$ gravity, which show in detail that scenario B) is realized in that case[117].

Although most results on $2+1$ gravity do not extend to the physical, $3+1$ theory, there is an argument for scenario $B$ that does appear to extend to $3+1$ [118]. This argument starts with the theory with non-zero cosmological constant. In $2+1$ gravity, with non-zero $\Lambda$, the symmetry group of boundary states is $S O_{q}(3,1)$, the quantum deformed deSitter group, with deformation parameter given in the $2+1$ dimensional case by

$$
\ln q \sim l_{P} \sqrt{\Lambda}
$$

When one takes the contraction in which the cosmological constant goes to zero one does 
not get, as usual the Poincare algebra. Instead, because $q \rightarrow 1$ as $\Lambda \rightarrow 0$ one gets a deformation of the Poincare algebra parameterized by the Planck length $l_{P}$. This is the $\kappa$ Poincare algebra, which is known to provided an example of Scenario B. The emergence of $\kappa$-Poincare symmetry in $2+1$ gravity is also confirmed in several detailed studies[117].

One can run the same argument in $3+1$ dimensions, but one must use the relation (48) instead of (50). Because the theory has local field degrees of freedom, one must also renormalize the energy and momentum generators by an appropriate power of $l_{P} \sqrt{\Lambda}$, which is the ratio of the physical ultraviolet and infrared cutoffs. When this is done, one recovers again the $\kappa$-deformation of the Poincare algebra[117].

Remarkably, evidence for the same result is found in studies of excitations of the Kodama state [11]. However, this result has been criticized because it is not known whether or not the Kodama state is a normalizable physical state. To counter this worry, the argument has been recast to show that it arises from studying excitations of a general semiclassical state[123].

\section{Frequently asked questions}

There is a set of questions often asked by physicists on first encountering loop quantum gravity. For the convenience of the readers, some of these are collected here, with answers.

1. How can there be a finite, well defined formulation of quantum general relativity when that theory is not normalizable in perturbation theory? The reason is that the standard perturbative approaches make two assumptions which are not made in the exact approach followed in LQG. i) Spacetime is smooth down to arbitrarily short distances, so there are physical degrees of freedom which propagate for arbitrarily high frequency and short wavelength. ii) The standard lorentz transformations correctly apply to these modes, no matter how high the frequency. Neither assumption could be made in a background independent approach. Indeed, the results of LQG falsify the first assumption and make testable the second. Physically speaking, there simply are no weakly coupled excitations of gravitational or matter fields with wavelength shorter than $l_{\text {Planck }}$.

2. Can the same methods be applied to ordinary quantum field theories? In fact some of the results of the loop representation were found first in lattice gauge theories, such as the fact that the spin networks give an orthonormal basis[165]. An approach to solving $Q C D$ numerically, with fermions, has been developed using the methods of loop quantum gravity[134]. However, as mentioned above, spin network states in the continuum give a non-separable Hilbert space, unless one mods out the state space by the action of the diffeomorphisms. Thus, they can be used in two contexts, either in the presence of a lattice regularization or in a gravitational theory in which the physical states are diffeomorphism invariant. 
3. Can LQG methods be applied to string theory? Background independent methods of quantization, analogous to LQG have been applied to the free string in [124, 125, 126]. These give theories which are well defined but which are unitarily inequivalent to the usual quantization of the free string. The reason is that the usual quantization uses a representation of $\operatorname{Dif} f\left(S^{1}\right)$ which is anomalous, whereas the LQGtype quantizations use non-anomalous representations. The former approach uses structure special to $1+1$ theories which is that there is a generator of $\operatorname{Diff}\left(S^{1}\right)$, called $L_{0}$, which in a certain gauge can be interpreted as the hamiltonian of the free string. It makes sense, in the quantization of a gauge fixed quantization, to choose a representation in which $L_{0}$ is positive and hermitian. The background independent quantizations on the other hand must treat all the generators of $\operatorname{Diff}\left(S^{1}\right)$ as constraints. Thus, the two methods are different and yield different theories. This does not mean one or the other is right, but it does illuminate an important difference between background independent and background dependent approaches to quantization.

4. LQG as well as finite dimensional models based on it studied in Loop Quantum Cosmology depend on a non-standard representation of the quantum theory in which the canonical commutation relations are not represented. Instead, one quantizes commutation relations involving exponentials of the basic variables. When this approach is applied to ordinary systems such as the harmonic oscillator it gives a theory that is unitarily inequivalent to the standard quantum theory. Why then should be trust it when applied to gravity? For field theories, the standard quantization, which leads to Fock space, depends on a fixed background metric. So it is simply unavailable for the case of a background independent approach to quantum gravity. A new approach is needed, which must be based on the quantization of a non-canonical algebra. This is what is done in loop quantum gravity. The fact that ordinary QFT is reproduced in suitable limits, as discussed in results above shows that the standard results of quantum theory are reproduced when suitable approximations are made, leading to the emergence of physics on a background spacetime.

5. Why isn't supersymmetry necessary, when string theory says it is for perturbative finiteness of quantum gravity? The answer is straightforward. Because there are no infinite sums over arbitrarily high energy modes, there is no need for supersymmetry to cancel them, by balancing the contributions of fermions and bosons. There are, so far, no results that indicate that matter context effects the consistency or finiteness of the quantum theory of gravity. Loop quantum gravity appears equally finite and consistent coupled to any matter fields. At the same time there is no problem extending all the main results to $N=1$ supergravity[32]-[35] There are interesting problems extending loop quantum gravity to $N=2$ and higher, which have yet to be tackled. 
6. Do you have anything to say about the problem of unification? There are arguments that matter is necessarily included in loop quantum gravity, as a result of mismatches between microsoiopic and macroscopic notions of locality[140] and topololgy[139].

7. Do you have anything to say about the problem of the cosmological constant?YES. There is a proposal for a mechanism to dynamically tune the cosmological constant to zero, analogous to the Pecci-Quinn mechanism[121]. It can also be mentioned that, unlike approaches that depend on supersymmetry, there is no special issue incorporating a finite cosmological constant of either sign into the theory. There is also an argument that the problem of the cosmological constant is a pseudo-problem brought on by background dependent approaches, brought on by an unphysical split of the fundamental degrees of freedom into two sets, which represent the background and excitations of the background[127].

8. What about the problem of the existence of the low energy limit? As described above, there is no problem studying the low energy limit using techniques analogous to those of conventional theories. One can easily write down candidates for the ground state, and show that they have several properties required for the ground state. For example, one can study small excitations of these states and recover ordinary quantum field theory on background manifolds, as well as the linearized graviton states, at least in the limit of long wavelength. The difficulties that remain with the low energy limit have mostly to do with finding criteria to distinguish the physically correct ground state from other candidates.

9. Can you calculate graviton-graviton scattering? In principle yes. One could easily study interactions among the excitations of candidate ground states such as those in $[101,102,105,106,11]$. The result must be finite as the discrete of area and volume impose an ultraviolet cutoff on the spectra of excitations of any candidate ground state. Presumably the results will depend on the properties of the ground state, and a crucial issue will be the fate of Lorentz invariance. We would not be surprised to see that the scattering of excitations around ground states of the form of [101] that depend on preferred frames break Lorentz invariance explicitly. Still, it is embarrassing that this has not been done for some examples, as it would be illuminating to see exactly how perturbative finiteness arises.

10. In string theory we take background independence to mean independent of the particular vacuum perturbative string theory is defined with respect to. Is this the same meaning you use in LQG? No, the key point in the definition given in section 2 above is that the quantum theory is completely defined without reference to any fixed background fields. This is different from a formulation that requires a background field, even if there may be many different background fields that will serve $\mathrm{e}^{32}$.

\footnotetext{
${ }^{32}$ For more on the meaning of background independence, see [128].
} 
11. What about the dimension and topology of the manifold. Shouldn't that be determined dynamically as well? The general philosophical principle that historically motivates background independence is the idea that space and time should be purely relational quantities. This does imply that one who believes that principle would prefer that topology and dimension are emergent and dynamically determined quantities. However, this is not the case in general relativity and other classical diffeomorphism invariant field theories. While one can study general relativity for different topologies, dimensions and choices of boundary conditions, each set of these choices defines a phase space. The different phase spaces corresponding to the different choices of dimension, topology and differential structure are disconnected from each other. To construct a phase space the topology must be chosen to be of the form of $\Sigma \times R$, with the spatial manifold $\Sigma$ fixed, hence topology change cannot be described in a canonical formalism. In a Hamiltonian quantization there is then a different Hilbert space for each choice of topology, dimension and boundary conditions, so these are fixed as well within each quantum theory ${ }^{33}$.

This circumstance does not prevent people from taking the structures from loop quantum gravity and proposing new theories in which the states and histories are defined in terms only of combinatorial graphs and representation theory, so that the dimension and topology may emerge as aspects of the low energy behavior. There might then be phase transitions in which topology and dimension change., Such a theory could not be strictly speaking the "quantization of a classical field theory", but some believe that makes it more rather than less likely to describe nature. These theories are mentioned in 4.8 above.

12. Could string theory and loop quantum gravity be different sides of the same theory? It is natural to make this suggestion as both string theory and loop quantum gravity realize the principle of duality-the former in a background dependent context and the latter in a background independent context. This makes it natural to hypothesize that excitations around background loop quantum gravity states whose semiclassical approximations look like smooth spacetimes should behave like perturbative strings, at least in a regime below the Planck scale. This possibility has been explored in a number of papers[36]. The results look intriguing but there is more to do. Given that string/ $\mathcal{M}$ theory needs a background independent formulation, and given that the most developed approach to quantum gravity at the background independent level is loop quantum gravity, it is natural to look for a version of loop quantum gravity that could serve as the background independent form of string theory. For example, one can study spin foam models based on the conjectured symmetries of $\mathcal{M}$ theory such as $O s p(1 \mid 32)$. One approach to unifying string theory and loop quantum gravity by subsuming both in a certain extension of a matrix formulation of Chern-Simons theory is described in [36].

\footnotetext{
${ }^{33}$ This issue is discussed in more detail in [128].
} 
13. I've heard that the Kodama state has been shown to be unphysical. Is this true? Some criticisms have been made concerning a related state in Yang-Mills theory, discussed by Jackiw in [114] and Wittin in [115]. It is likely that this state is unphysical in the Hilbert space of Yang-Mills theory for the reasons discussed by those authors. However the analogy to quantum general relativity is not very helpful here, for reasons discussed in [116]. For one thing, the Yang-Mills version does not play the role of a semiclassical state to describe excitations around the classical ground state, as is the case in gravity. The precise situation is as was described in result 27 above. The Kodama state is an exact solution to the constraints of quantum gravity and is also a semiclassical state corresponding to an expansion around de Sitter (or AdS) spacetime[109]. It is not known whether or not the Kodama state is normalizable in the physical inner product. A naive truncation results in a linearized state that is not normalizable in the lorentzian case and delta-functional normalizable in the Euclidean case[116]. Semiclassical expansions around the Kodama state lead to a recovery of QFT on deSitter (or AdS) spacetimes[110, 11].

14. Does loop quantum gravity have anything to say about the holographic principle? Quite a bit. It should be mentioned that Crane, in [172] made a conjecture for a background independent formulation of the holographic principle, a bit before the papers of ' $t$ Hooft[173] and Susskind[174]. Crane's formulation inspired the study of ChernSimons boundary terms[16], which then led to the studies of black hole entropy. In fact, the $N$-bound discussed in [175] can be explained in terms of loop quantum gravity, as discussed in [11]. A background independent formulation of the holographic principle, suitable for theories in which the spacetime geometry is fully quantized was proposed in [176] and compared to other formulations in [177].

15. Isn't it true that the Ashtekar-Sen connection lives in a complex extension of $S U(2)$ ? Doesn't this mean that the inner product cannot be defined so that one has just traded one set of problems for another? This is wrong on two counts. First, most results listed above are based on the use of the Barbero connection, which is valued in the real $S U(2)$. The inner products on $\mathcal{H}^{\text {kin }}$ and $\mathcal{H}^{\text {diffeo }}$ are then rigorously defined and a full normalizable basis is known explicitly in each case. Thiemann's form of the Hamiltonian constraint applies to this case (for both the Lorentzian and Euclidean signature.) The Hamiltonian constraint is finite and well defined, acting on $\mathcal{H}^{\text {diffeo }}$ and an infinite number of exact solutions are known.

There continues to be some interest in the Ashtekar-Sen connection. Not as many rigorous results have been achieved for it, but the problem is not that of trying to make a Schroedinger-like representation on a space with a non-compact gauge field. For example., there is no problem at all working out the details of the linearized theory of spin-two fields in terms of the linearization of the Ashtekar connection[103, 116]. The reason is that the Ashtekar-Sen connection is a non-linear version of a Bargmann coordinate, $z=q+i p$ for an ordinary quantum system. The states are 
then holomorphic functionals of $A_{a}^{i}$. The key open problems concern the normalizability of such states under the physical inner product. So far it has been easier to address such problems in the context of the Barbero connection, but the problem remains open.

16. I've heard there is some problem with the consistency of the Hamiltonian constraints[166]. What was it, and has it been resolved? There is no problem with the consistency of the constraints. To explain why is a bit technical, but given the importance of the issue I will go into it. The issue has to do with how the classical algebra of the constraints of general relativity is realized quantum mechanically[167, 168]. Classically, the Poisson brackets of two Hamiltonian constraints, $H(x)$, of the form of (39) have the form

$$
\{H(x), H(y)\}=\partial_{a} \delta^{3}(x, y) q q^{a b} D_{b}
$$

where $D_{b}$ is the diffeomorphism constraint, (38). The problem is that this is not a Lie algebra because the structure constant of the algebra includes $q^{a b}$, a dynamical variable $^{34}$. The problem is then what happens to the algebra in the quantum theory? There are several aspects to this problem. First, the operators are all regularized in the quantum theory, so $H(x)$ is realized by a one parameter family $\hat{H}_{\epsilon}$. The condition that a state be physical is that

$$
\lim _{\epsilon \rightarrow 0}\left(\hat{H}_{\epsilon}(x) \Psi\right)=0 .
$$

This limit must be taken in a suitable norm, which turns out to be the inner product of the gauge but not diffeomorphism invariant states (because the regulated $\hat{H}_{\epsilon}$ is not diffeomorphism covariant for finite $\epsilon$, and only becomes so in the limit $\epsilon \rightarrow 0$.)

For the theory to exist, there must be an infinite number of simultaneous solutions to (52) which also live in the space $H^{\text {diffeo }}$ of diffeomorphism invariant states. In fact, there are, given a completely explicit construction of $\hat{H}_{\epsilon}$, infinite numbers of such solutions have been constructed explicitly.

The question is then what is the right hand side of,

$$
\left[\hat{H}_{\epsilon}(x), \hat{H}_{\epsilon^{\prime}}(y)\right]=?
$$

From the existence of an infinite number of solutions to (52) no anomalous c-number term can appear on the right hand side. So the algebra is consistent.

In general, what appears on the right hand side depends on the regularization and ordering studied. In the most studied approach, due to Thiemann, the right hand side vanishes in the limit, when evaluated on diffeomorphism invariant states. This of course agrees with the expectation from (51) and it is enough to ensure the consistency of the theory. However, a simple, but quantum mechanical form of (51) is

\footnotetext{
${ }^{34}$ This is sometimes referred to as a business class, rather than a first class algebra.
} 
not easily obtained in the space of kinematical states $\mathcal{H}^{k i n}$. Whether or not this is a problem is not clear.

In other regularizations, such as that studied originally in [69], the right hand side appears formally in the order $\hat{D}_{a} \hat{q}^{a b}$. This makes it possible to find solutions to (52) which are not diffeomorphism invariant. Instead they satisfy $\hat{D}_{a} \hat{q}^{a b} \Psi=0$. This turns out to mean that the states need only be invariant under diffeomorphisms tangent to the loops, which they are by virtue of the reparameterization invariance of Wilson loops.

What is important is that there is no anomaly or inconsistency. A few practitioners would still prefer a regularization and ordering such that the right hand side (53) reproduced a term non-vanishing in the limit with the ordering $\hat{q}^{a b} \hat{D}_{a}$. This would of course imply that only diffeomorphism invariant states could satisfy (52), (when integrated over all smooth test functions.) So far none is known. However, there is not to my knowledge a compelling case for requiring this. What is necessary, is to insure that the theory has propagation of physical degrees of freedom. One way to ask this is the following: Are there solutions to the full set of constraints which describe gravitons propagating on classical backgrounds? If so the theory contains the quantization of the physical degrees of freedom of general relativity. Examples of such solutions are described in [11].

Another response to this issue has been to suggest that there is no need for a theory of quantum gravity to have a generator of infinitesimal time evolution if it has generators of finite evolution. Indeed, given that there is on $\mathcal{H}^{k i n}$ no generator of infinitesimal spatial diffeomorphisms, but only a generator of finite diffeomorphisms, it would contradict relativity were there to be a well defined generator of infinitesimal time translations. This argument was one of the basic motivations for the invention of spin foam models and justifies the inclusion into the spin foam models of moves which are not present in Thiemann's constraint $[53,58]$.

\section{Open questions in loop quantum gravity}

I close with a brief list of some problems of current interest.

- There is an urgency to determine what unambiguous predictions can be made for the results of upcoming experiments on Planck scale phenomenology.

- A similar situation holds for soon to be measured features of the CMB spectrum, such as polarization and the tensor modes.

- Gravitons and other quanta are known to arise as weakly coupled excitations of several different ansatzes for the ground state. As there are no modes below the Planck length, the scattering of these excitations must be computable and finite. It would 
be good to calculate some examples of scattering and confirm this expectation value in detail. The answer will of course depend on the ansatz chosen for the ground state.

- In contexts with a boundary, where there is a classical positive definite energy, is there an expression for the expectation value of the energy which is positive definite, thus extending to the quantum theory the positive energy theorem?

- Were there such a positive definite expression for the energy, one could determine which is the right ansatz for the ground state by minimizing it, either exactly or approximately. This would lead to unambiguous predictions for Planck scale phenomenology. An alternative way to do this would be to establish a general result about the symmetry of the ground state, i.e. is it invariant under the ordinary or deformed Poincare algebra? This would also lead to predictions for scattering of gravitons and other quanta.

- In conventional QFT we have learned that good theories are associated with critical phenomena in statistical system, connected to the path integral by Euclidean continuation. Given that euclidean continuation is not necessarily available in quantum gravity, one can ask whether there is a characteristic critical phenomena associated with quantum gravitational systems[129].

- There are indications that LQG contains matter degrees of freedom intrinsically, so they do not have to be put in, which need more development[140, 141, 139].

- Dreyer's work concerning the relationship between black hole entropy and quasi normal modes[84] is reassuring, because it shows that the same value of the Imirzi parameter (or finite renormalization of Newton's constant) suffices to match both properties of black holes. But it leads to a puzzle: does this mean that loop quantum gravity with the parameter chosen differently cannot have a good classical limit? Is the correct value an attractor or a fixed point?

- Loop quantum gravity can be extended to incorporate supersymmetry. But is there anything that supersymmetry does for quantum gravity at a background independent level, that would lead to a reason to prefer a supersymmetric theory?

- Loop quantum gravity has its simplest formulation in terms of the chiral AshtekarSen variables. The classical theory is nevertheless chirally symmetric, but it was suggested a long time ago by Soo[111] that there might be violations of $\mathrm{CP}$ and chirally asymmetric effects quantum mechanically, and evidence for such effects was found in studies of one ansatz for the ground state[22]. It would be good to know if LQG makes unambiguous predictions about chiral symmetry breaking and $C P^{35}$.

\footnotetext{
${ }^{35}$ Some recent results about this are in [130].
} 
- There are some technical problems remaining in establishing the detailed connection between the Hamiltonian and path integral, or spin foam, formulations. There are similarly technical issues related to the exact relationship between the different approaches to spin foam models.

- It is known that dynamical triangulation models have no good continuum limit in the case of 4, Euclidean dimensions[38], but there is recent evidence that a lorentzian variant has a good low energy limit in $3+1$ dimensions [39, 43]. One can conjecture that the same is true for spin foam models, i.e. that there will be a good low energy limit only in the causal or lorentzian case. To show this one might start by adding labels to CDT models, so as to turn them into spin foam models. It will be also necessary to extend the CDT models so that they do not depend on a rigid time slicing. This seems to be possible, at least in $1+1[169$ ] and $2+1$ dimensions[170]. A useful step in this direction is also to re-express the CDT models as spin systems[171]

- While the physical inner product ${ }^{36}$ is known formally to be expressed as a path integral in the spin foam language, it would be good to have it in a more explicit form. This would allow us to check whether physical states such as the Kodama state are normalizable in the exact physical inner product.

- More work needs to be done on the renormalization group for spin foam models[107, 108].

- There remain foundational issues having to do with the wavefunction of the universe. The fact that we can do quantum cosmology in detail, with real local degrees of freedom, rather than just study models, makes the resolution of these issues more urgent. Loop quantum gravity has stimulated new ideas in this direction, such as quantum causal histories $[54,55]$ and a proposal that spin networks might underlie a theory of hidden variables[142].

\section{Conclusions}

The spirit of this review has been to lay out the results so that the reader can draw their own conclusions. In case it is interesting, the author would summarize the present state as knowledge as follows.

The open problems are non-trivial and the results so far by no means are sufficient to show that loop quantum gravity is the right theory of quantum gravity. But the results are sufficient to say that diffeomorphism invariant quantum gauge field theories exist, rigorously, in both canonical and path integral form. The resulting theories are background independent, as any plausible quantum theory of spacetime must be. A powerful toolkit

\footnotetext{
${ }^{36}$ By which is meant the inner product on the subset of states that satisfy all the constraints. The inner product on spatially diffeomorphism invariant states is known in closed form.
} 
of calculational techniques has been developed and many non trivial, and in some cases, surprising results have been found. Among these theories are the quantization of general relativity coupled to the standard matter fields and supergravity.

These theories are finite quantum field theories and the remaining open problems concern, not their existence, but whether they reproduce general relativity correctly in the low energy limit and make correct predictions for experiments that probe beyond that limit. This is to say that loop quantum gravity is now part of ordinary physics in that future progress will result from refinement of calculational techniques and models and comparisons of the results gotten with experiment. It is possible, but by no means certain, that the result of this process will be an accumulation of evidence that loop quantum gravity correctly describes the physics of the Planck scale.

While other approaches to quantum gravity also have gotten impressive results, there is not another approach to quantum gravity about which all of the foregoing can be said ${ }^{37}$, or about which there is such a long list of robust results concerning Planck scale physics.

There is much still to do, and the sentiment expressed by the title is genuine. Loop quantum gravity may still not be right. But it can, perhaps, be said objectively that it is at least, among the approaches so far known, the most reasonable point of departure for the discovery of the right theory of quantum gravity.

\section{Acknowledgments}

I must thank my collaborators over the years who have taught me most of what I know about quantum gravity, Stephon Alexander, Matthias Arnsdorf, Abhay Ashtekar, Roumen Borissov, Louis Crane, John Dell, Ted Jacobson, Laurent Freidel, Jurek Kowalski-Glikman, Yi Ling, Seth Major, Joao Magueijo, Justin Malecki, Fotini Markopoulou, Carlo Rovelli, Chopin Soo and Artem Starodubtsev. Conversations with many other people during the preparation of this review have been very helpful, especially Jan Ambjorn, Olaf Dreyer, Renate Loll and Thomas Thiemann. I am grateful as well to the NSF and the Phillips Foundation for their very generous support which made my own work possible. I am thankful especially to my colleagues at Perimeter Institute for many critical discussions. Finally, I am grateful to John Schwarz for pointing out the need for this review, and to Thomas Thiemann for a careful and critical reading of the manuscript.

\section{References}

[1] A. Sen, On the existence of neutrino zero modes in vacuum spacetime J. Math. Phys. 22 (1981) 1781, Gravity as a spin system Phys. Lett. B11 (1982) 89.

\footnotetext{
${ }^{37}$ The best developed alternative, string theory, has also impressive results, but those concerning the consistent unification of general relativity with quantum theory are limited so far to second order (genus two) perturbation theory around fixed backgrounds[12].
} 
[2] Abhay Ashtekar, New variables for classical and quantum gravity," Phys. Rev. Lett. 57(18), 2244-2247 (1986).

[3] C. Rovelli, Notes for a brief history of quantum gravity, gr-qc/0006061.

[4] A. Ashtekar, New perspectives in canonical gravity (Bibliopolis, Naples, 1988); Lectures on non-perturbative canonical gravity, Advanced Series in Astrophysics and Cosmology-Vol. 6 (World Scientific, Singapore, 1991).

[5] R. Gambini and J. Pullin, Loops, knots, gauge theories and quantum gravity Cambridge University Press, 1996

[6] C. Rovelli, Quantum Gravity, Cambridge University Press, 2004.

[7] Thomas Thiemann, "Introduction to Modern Canonical Quantum General Relativity" gr-qc/0110034, Cambridge University Press, to appear.

[8] A. Ashtekar and J. Lewandowski, Background independent quantum gravity: a status report, gr-qc/0404018.

[9] C. Rovelli, Living Rev. Rel. 1 (1998) 1, gr-qc/9710008.

[10] L. Smolin: in Quantum Gravity and Cosmology, eds J Perez-Mercader et al, World Scientific, Singapore 1992; The future of spin networks gr-qc/9702030 in the Penrose Festschrift.

[11] L. Smolin, Quantum gravity with a positive cosmological constant, hep-th/0209079.

[12] L. Smolin, How far are we from the quantumtheory of gravity? , hep-th/0303185.

[13] J. Baez, Spin foam models, Class. Quant. Grav. 15 (1998) 1827-1858, gr-qc/9709052; An introduction to spin foam models of quantum gravity and BF theory. Lect.Notes Phys., 543:25 94, 2000.

[14] J. Iwasaki, A reformulation of the Ponzano-Regge quantum gravity model in terms of surfaces," gr-qc/9410010; A definition of the Ponzano-Regge quantum gravity model in terms of surfaces," gr-qc/9505043, J. Math. Phys. 36 (1995) 6288; L. Freidel and K. Krasnov. Spin foam models and the classical action principle. Adv.Theor.Math.Phys., 2:11831247, 1999;

[15] A. Perez, Spin Foam Models for Quantum Gravity, Topical Review in Class.Quant.Grav. 20 (2003) R43 gr-qc/0301113.

[16] L. Smolin, Linking topological quantum field theory and nonperturbative quantum gravity, J. Math. Phys. 36(1995)6417, gr-qc/9505028. 
[17] K. Krasnov, On Quantum Statistical Mechanics of a Schwarzschild Black Hole , grqc/9605047, Gen. Rel. Grav. 30 (1998) 53-68; C. Rovelli, “Black hole entropy from loop quantum gravity," grqc/9603063.

[18] A. Ashtekar, J. Baez, K. Krasnov, Quantum Geometry of Isolated Horizons and Black Hole Entropy gr-qc/0005126; A. Ashtekar, J. Baez, A. Corichi, K. Krasnov, “Quantum geometry and black hole entropy," gr-qc/9710007, Phys.Rev.Lett. 80 (1998) 904-907.

[19] J. D. Bekenstein, Lett. Nuovo. Cim 11(1974).

[20] Martin Bojowald,"Isotropic Loop Quantum Cosmology", Class.Quant.Grav. 19 (2002) 2717-2742, gr-qc/0202077; "Inflation from Quantum Geometry", grqc/0206054; "The Semiclassical Limit of Loop Quantum Cosmology", gr-qc/0105113, Class.Quant.Grav. 18 (2001) L109-L116; “ Dynamical Initial Conditions in Quantum Cosmology", gr-qc/0104072, Phys.Rev.Lett. 87 (2001) 121301; Absence of Singularity in Loop Quantum Cosmology, gr-qc/0102069, Phys.Rev.Lett. 86 (2001) 5227.

[21] S. Tsujikawa, P. Singh, R. Maartens, Loop quantum gravity effects on inflation and the $C M B$, astro-ph/0311015

[22] Rodolfo Gambini, Jorge Pullin, "Nonstandard optics from quantum spacetime", Phys.Rev. D59 (1999) 124021, gr-qc/9809038;

[23] Jorge Alfaro, Hugo A. Morales-Tcotl, Luis F. Urrutia, "Loop quantum gravity and light propagation", Phys.Rev. D65 (2002) 103509, hep-th/0108061.

[24] T. T. Wu and C. N. Yang, Concept Of Nonintegrable Phase Factors And Global Formulation Of Gauge Fields, Phys. Rev. D 12, 3845 (1975).

[25] P. A. M. Dirac, Lectures on Quantum Mechanics Belfer Graduate School of Science Monographs, no. 2 (Yeshiva University Press, New York,1964).

[26] J. Stachel, "Einstein's search for general covariance, 1912-15" in Einstein and the History of General Relativity vol 1 of Einstein Studies eds. D. Howard and J. Stachel. (Birkhauser,Boston,1989).

[27] See, for example, Earman, J. 1989. World Enough and Spacetime: Absolute vs. Relational Theories of Space and Time. Cambridge, MA: MIT Press; Norton, J. D. 1987. Einstein, the Hole Argument and the Reality of Space. In J. Forge (ed.), Measurement, Realism and Objectivity, 153-188. Boston: D. Reidel; L. Smolin, "The present moment in quantum cosmology: Challenges to the arguments for the elimination of time," arXiv:grqc/0104097.

[28] Julian Barbour, The End of Time, Oxford University Press, 2000; “Leibnizian Time, Machian Dynamics, And Quantum Gravity," In *Oxford 1984, Proceedings, Quantum Concepts In Space and Time*, 236-246. 
[29] C. Rovelli, "What Is Observable In Classical And Quantum Gravity?," Class. Quant. Grav. 8, 297 (1991).

[30] L. Smolin Life of the Cosmos, (Oxford University Press, New York and Wiedenfeld and Nicolson, London, 1997).

[31] J. Lewandowski, A. Okolow, H. Sahlmann, T. Thiemann, Uniqueness of diffeomorphism invariant states on holonomy-flux algebras, gr-qc/0504147; Christian Fleischhack, Representations of the Weyl Algebra in Quantum Geometry math-ph/0407006; Hanno Sahlmann, SOME COMMENTS ON THE REPRESENTATION THEORY OF THE ALGEBRA UNDERLYING LOOP QUANTUM GRAVITY., gr-qc/0207111; WHEN DO MEASURES ON THE SPACE OF CONNECTIONS SUPPORT THE TRIAD OPERATORS OF LOOP QUANTUM GRAVITY? , gr-qc/0207112; H. Sahlmann and T. Thiemann, ON THE SUPERSELECTION THEORY OF THE WEYL ALGEBRA FOR DIFFEOMORPHISM INVARIANT QUANTUM GAUGE THEORIES. , grqc/0302090; IRREDUCIBILITY OF THE ASHTEKAR-ISHAM-LEWANDOWSKI REPRESENTATION., gr-qc/0303074; A. Okolow and J. Lewandowski DIFFEOMORPHISM COVARIANT REPRESENTATIONS OF THE HOLONOMY FLUX *ALGEBRA., Class.Quant.Grav.20:3543-3568,2003, gr-qc/0302059;

[32] Y. Ling and L. Smolin, Supersymmetric spin networks and quantum supergravity, Phys. Rev. D61, 044008(2000), hep-th/9904016; Holographic Formulation of Quantum Supergravity, hep-th/0009018, Phys.Rev. D63 (2001) 064010.

[33] T. Jacobson, New Variables for canonical supergravity, Class. Quant. Grav.5(1988)923- 935; D. Armand-Ugon, R. Gambini, O. Obregon, J. Pullin, Towards a loop representation for quantum canonical supergravity, hep-th/9508036, Nucl. Phys. B460 (1996) 615; L. F. Urrutia Towards a loop representation of connection theories defined over a super-lie algebra, hep-th/9609010; H. Kunitomo and T. Sano The Ashtekar formulation for canonical N=2 supergravity, Prog. Theor. Phys. suppl. (1993) 31; Takashi Sano and J. Shiraishi, The Nonperturbative Canonical Quantization of the N=1 Supergravity, Nucl. Phys. B410 (1993) 423, hep-th/9211104; The Ashtekar Formalism and WKB Wave Functions of N=1,2 Supergravities, hep-th/9211103; T. Kadoyoshi and S. Nojiri, $\mathrm{N}=3$ and $\mathrm{N}=4$ two form supergravities, Mod. Phys. Lett. A12:1165-1174,1997, hep-th/9703149; K. Ezawa, Ashtekar's formulation for N=1, $\mathrm{N}=2$ supergravities as constrained BF theories, Prog. Theor. Phys.95:863-882, 1996, hep-th/9511047.

[34] Yi Ling, "Introduction to supersymmetric spin networks", hep-th/0009020, J.Math.Phys. 43 (2002) 154-169

[35] Y. Ling and L. Smolin, "Eleven dimensional supergravity as a constrained topological field theory," Nucl. Phys. B 601, 191 (2001) [arXiv:hep-th/0003285]; L. Smolin, 
"Chern-Simons theory in 11 dimensions as a non-perturbative phase of $\mathrm{M}$ theory," arXiv:hep-th/9703174.

[36] Lee Smolin,Strings as perturbations of evolving spin-networks, Nucl.Phys.Proc.Suppl. 88 (2000) 103-113, hep-th/9801022; "M theory as a matrix extension of Chern-Simons theory", hep-th/0002009, Nucl.Phys. B591 (2000) 227-242; "The cubic matrix model and a duality between strings and loops", hep-th/0006137; "The exceptional Jordan algebra and the matrix string", hep-th/0104050; Takehiro Azuma, Maxime Bagnoud, Curvedspace classical solutions of a massive supermatrix model, hep-th/0209057; Maxime Bagnoud, Luca Carlevaro, Adel Bilal, Supermatrix models for M-theory based on osp(1-32,R), Nucl.Phys.B641:61-92,2002, hep-th/0201183; Takehiro Azuma, Satoshi Iso, Hikaru Kawai, Yuhi Ohwashi, Supermatrix Models, Nucl.Phys. B610 (2001) 251279, hep-th/0102168; Takehiro Azuma, Investigation of Matrix Theory via Super Lie Algebra, hep-th/0103003.

[37] L. Bombelli, J. H. Lee, D. Meyer and R. Sorkin, "Space-Time As A Causal Set," Phys. Rev. Lett. 59, 521 (1987); X. Martin, D. O'Connor, D. P. Rideout and R. D. Sorkin, "On the 'renormalization' transformations induced by cycles of expansion and contraction in causal set cosmology," Phys. Rev. D 63, 084026 (2001) [arXiv:gr-qc/0009063]; D. P. Rideout and R. D. Sorkin, "Evidence for a continuum limit in causal set dynamics," Phys. Rev. D 63, 104011 (2001) [arXiv:gr-qc/0003117]; David Rideout, Dynamics of Causal Sets, gr-qc/0212064; D. P. Rideout, R. D. Sorkin, A Classical Sequential Growth Dynamics for Causal Sets, gr-qc/9904062, Phys.Rev. D61 (2000) 024002.

[38] J. Ambjorn, Z. Burda, J. Jurkiewicz and C. F. Kristjansen, “Quantum gravity represented as dynamical triangulations," Acta Phys. Polon. B 23, 991 (1992); J. Ambjorn, "Quantum Gravity Represented As Dynamical Triangulations," Class. Quant. Grav. 12, 2079 (1995); M. E. Agishtein and A. A. Migdal, "Simulations of four-dimensional simplicial quantum gravity," Mod. Phys. Lett. A 7, 1039 (1992).

[39] J. Ambjorn, A. Dasgupta, J. Jurkiewiczcy and R. Loll, “A Lorentzian cure for Euclidean troubles", hep-th/0201104; J. Ambjorn and R. Loll, Nucl. Phys. B536 (1998) 407 [hep-th/9805108]; J. Ambjorn, J. Jurkiewicz and R. Loll, Phys. Rev. Lett. 85 (2000) 924 [hepth/ 0002050]; Nucl. Phys. B610 (2001) 347 [hep-th/0105267]; R. Loll, Nucl. Phys. B (Proc. Suppl.) 94 (2001) 96 [hep-th/0011194]; J. Ambjorn, J. Jurkiewicz and R. Loll, Phys. Rev. D64 (2001) 044011 [hep-th/0011276]; J. Ambjorn, J. Jurkiewicz, R. Loll and G. Vernizzi, Lorentzian $3 d$ gravity with wormholes via matrix models, JHEP 0109, 022 (2001) [arXiv:hep-th/0106082]; B. Dittrich (AEI, Golm), R. Loll, A Hexagon Model for 3D Lorentzian Quantum Cosmology, hep-th/0204210.

[40] Rodolfo Gambini, Jorge Pullin, Canonical quantization of general relativity in discrete space-times , gr-qc/0206055, Phys.Rev.Lett. 90 (2003) 021301; [abs, ps, pdf, other] : Discrete quantum gravity: applications to cosmology, gr-qc/0212033, Class.Quant.Grav. 20 (2003) 3341; Canonical quantum gravity and consistent discretizations, gr-qc/0402062; R. 
Gambini, R. Porto, J. Pullin Consistent discrete gravity solution of the problem of time: a model gr-qc/0302064

[41] A. Dasgupta and R. Loll, Nucl. Phys. B606 (2001) 357 [hep-th/0103186].

[42] J. Ambjorn (Niels Bohr Institute), K.N. Anagnostopoulos (Univ. of Crete), R. Loll, Crossing the $c=1$ barrier in $2 d$ Lorentzian quantum gravity, hep-lat/9909129, Phys.Rev. D61 (2000) 044010.

[43] J. Ambjorn, J. Jurkiewiczcy and R. Loll, Emergence of a 4D World from Causal Quantum Gravity, hep-th/0404156.

[44] W. Fairbairn, C. Rovelli, Separable Hilbert space in Loop Quantum Gravity, grqc/0403047.

[45] J.F. Plebanski. On the separation of einsteinian substructures. J. Math. Phys., 18:2511, 1977.

[46] T. Jacobson and L. Smolin, Phys. Lett. B 196 (1987) 39; Class. and Quant. Grav. 5 (1988) 583; J. Samuel, Pramana-J Phys. 28 (1987) L429.

[47] R. Capovilla, J. Dell and T. Jacobson, Phys. Rev. Lett. 21, 2325(1989); Class. Quant. Grav. 8, 59(1991); R. Capovilla, J. Dell, T. Jacobson and L. Mason, Class. and Quant. Grav. 8, 41(1991).

[48] J. Barbero, Real Ashtekar variables for Lorentzian signature spacetime," Phys. Rev. D51 (1995) 5507.

[49] T. Thiemann, "Quantum spin dynamics," gr-qc/9606089, gr-qc/9606090; "Anomaly free formulation of non-perturbative 4-dimensional Lorentzian quantum gravity," Phys. Lett. B380 (1996) 257.

[50] L. Smolin, A holograpic formulation of quantum general relativity, Phys. Rev. D61 (2000) 084007, hep-th/9808191.

[51] A. Ashtekar, V. Husain, C. Rovelli, J. Samuel and L. Smolin 2+1 quantum gravity as a toy model for the 3+1 theory Class. and Quantum Grav. L185- L193 (1989); L. Smolin, Loop representation for quantum gravity in $2+1$ dimensions, in the proceedings of the John's Hopkins Conference on Knots, Tolopoly and Quantum Field Theory ed. L. Lusanna (World Scientific,Singapore,1989) .

[52] L. Freidel, K. Krasnov, R. Puzio, “ BF Description of Higher-Dimensional Gravity Theories", hep-th/9901069, Adv.Theor.Math.Phys. 3 (1999) 1289-1324.

[53] Fotini Markopoulou, "Dual formulation of spin network evolution", gr-qc/9704013.

[54] F. Markopoulou, Planck-scale models of the Universe, gr-qc/0210086. 
[55] E. Hawkins, F. Markopoulou, H. Sahlmann, Evolution in Quantum Causal Histories, hep-th/0302111.

[56] Fotini Markopoulou, Lee Smolin, "Quantum geometry with intrinsic local causality", Phys.Rev. D58 (1998) 084032, gr-qc/9712067.

[57] Fotini Markopoulou, Lee Smolin, Causal evolution of spin networks, gr-qc/9702025, Nucl.Phys. B508 (1997) 409-430.

[58] 8] M. P. Reisenberger and C. Rovelli. Sum-over-surface form of loop quantum gravity," gr-qc/9612035, Phys. Rev. D 56 (1997) 3490; Spacetime as a Feynman diagram: the connection formulation. Class.Quant.Grav., 18:121140, 2001; Spin foams as Feynman diagrams, gr-qc/0002083.

[59] R. De Pietri, L. Freidel, K. Krasnov, and C. Rovelli. Barrett-Crane model from a boulatov-ooguri field theory over a homogeneous space. Nucl.Phys. B, 574:785806, 2000, hep-th/9907154.

[60] M. P. Reisenberger. "Worldsheet formulations of gauge theories and gravity," in Proceedings of the 7th Marcel Grossman Meeting, ed. by R. Jantzen and G. MacKeiser, World Scientific, 1996; gr-qc/9412035; A lattice worldsheet sum for 4-d Euclidean general relativity. gr-qc/9711052.

[61] J. Barrett and L. Crane, "Relativistic spin networks and quantum gravity", J. Math. Phys. 39 (1998) 3296-3302, gr-qc/9709028.

[62] R. Penrose, Theory of quantized directions unpublished manuscript; in Quantum theory and beyond ed T Bastin, Cambridge U Press 1971, in Advances in Twistor Theory, ed. L. P. Hughston and R. S. Ward, (Pitman,1979) p. 301in Combinatorial Mathematics and its Application (ed. D. J. A. Welsh) (Academic Press,1971).

[63] C. Rovelli and L. Smolin, Spin networks and quantum gravity, gr-qc/9505006, Physical Review D 52 (1995) 5743-5759.

[64] C. Rovelli and L. Smolin Discreteness of area and volume in quantum gravity, Nuclear Physics B 442 (1995) 593. Erratum: Nucl. Phys. B 456 (1995) 734; L. Smolin, "Finite Diffeomorphism Invariant Observables In Quantum Gravity," Phys. Rev. D 49, 4028 (1994) [arXiv:gr-qc/9302011].

[65] Roberto De Pietri and Carlo Rovelli, Geometry eigenvalues and the scalar product from recoupling theory in loop quantum gravity," Phys. Rev. D 54(4), 2664-2690 (1996); S. Fittelli, L. Lehner, C. Rovelli, The complete spectrum of the area from recoupling theory in loop quantum gravity," Class. Quant. Grav. 13, 2921-2932 (1996); Abhay Ashtekar and Jerzy Lewandowski, Quantum Theory of Geometry I: Area operators," Class. Quant. Grav. 14, A55-A81 (1997); T. Thiemann, -it Closed formula for the 
matrix elements of the volume operator in canonical quantum gravity, J.Math.Phys. 39 (1998) 3347-3371, gr-qc/9606091.

[66] R. Loll, "Spectrum of the Volume Operator in Quantum Gravity," Nucl. Phys. B 460, 143 (1996) [arXiv:gr-qc/9511030]; “The Volume operator in discretized quantum gravity," Phys. Rev. Lett. 75, 3048 (1995) [arXiv:gr-qc/9506014].

[67] J. Brunnemann, T. Thiemann, Simplification of the Spectral Analysis of the Volume Operator in Loop Quantum Gravity, gr-qc/0405060

[68] L. Smolin, Finite, diffeomorphism invariant observables in quantum gravity, Phys. Rev. D 49 (1994) 4028, gr-qc/9302011.

[69] T. Jacobson and L. Smolin, Nucl. Phys. B 299 (1988) 295.

[70] C. Rovelli and L. Smolin, Knot theory and quantum theory, Phys. Rev. Lett 61(1988)1155; Loop representation of quantum general relativity, Nucl. Phys. B331(1990)80-152.

[71] R. Gambini and A. Trias, Phys. Rev. D23 (1981) 553, Lett. al Nuovo Cimento 38 (1983) 497; Phys. Rev. Lett. 53 (1984) 2359; Nucl. Phys. B278 (1986) 436; R. Gambini, L. Leal and A. Trias, Phys. Rev. D39 (1989) 3127.

[72] A Ashtekar J Lewandowski D Marlof J Mourau T Thiemann:"Quantization of dieomorphism invariant theories of connections with local degrees of freedom", grqc/9504018, JMP 36 (1995) 519.

[73] S. A. Major, “Operators for quantized directions," Class. Quant. Grav. 16, 3859 (1999) [arXiv:gr-qc/9905019]; "New operators for spin net gravity: Definitions and consequences," arXiv:gr-qc/0101032.

[74] T. Thiemann, A length operator for canonical quantum gravity, gr-qc/9606092, J.Math.Phys. 39 (1998) 3372-3392.

[75] L. Smolin, Time, measurement and information loss in quantum cosmology, grqc/9301016.

[76] C. Rovelli and L. Smolin, The physical hamiltonian in nonperturbative quantum gravity, Phys. Rev. Lett.72(1994)446; Spin Networks and Quantum Gravity, Phys. Rev. D52(1995)5743-5759.

[77] R. Borissov, Graphical Evolution of Spin Network States, Phys.Rev. D55 (1997) 60996111, gr-qc/9606013.

[78] T. Thiemann, Quantum Spin Dynamics (QSD) I \& II, Class.Quant.Grav. 15 (1998) 839-905, gr-qc/9606089, gr-qc/9606090. 
[79] T. Thiemann, personal communication.

[80] Alejandro Perez, Finiteness of a spinfoam model for euclidean quantum general relativity, Nucl.Phys. B599 (2001) 427-434, gr-qc/0011058 ; Louis Crane, Alejandro Perez, Carlo Rovelli, A finiteness proof for the Lorentzian state sum spinfoam model for quantum general relativity, gr-qc/0104057.

[81] John C. Baez, J. Daniel Christensen, Positivity of Spin Foam Amplitudes, Class.Quant.Grav. 19 (2002) 2291-2306, grqc/0110044; John C. Baez, J. Daniel Christensen, Greg Egan, Asymptotics of 10j symbols , gr-qc/0208010.

[82] L. Freidel and David Louapre, Non-perturbative summation over 3D discrete topologies, hep-th/0211026.

[83] L. Freidel, D. Louapre, Ponzano-Regge model revisited I: Gauge fixing, observables and interacting spinning particles, hep-th/0401076.

[84] O. Dreyer, Quasinormal Modes, the Area Spectrum, and Black Hole Entropy, grqc/0211076, Phys.Rev.Lett. 90 (2003) 081301.

[85] L. Motl, An analytical computation of asymptotic Schwarzschild quasinormal frequencies, gr-qc/0212096; L. Motl, A. Neitzke, Asymptotic black hole quasinormal frequencies, hepth/0301173.

[86] S. Alexandrov, On the counting of black hole states in loop quantum gravity, grqc/0408033; O. Dreyer, F. Markopoulou and L. Smolin, Symmetry and entropy of black hole horizons hep-th/0409056

[87] Krzysztof A. Meissner, Black hole entropy in Loop Quantum Gravity , gr-qc/0407052; Marcin Domagala, Jerzy Lewandowski, Black hole entropy from Quantum Geometry, gr-qc/0407051.

[88] Leonardo Modesto, Disappearance of Black Hole Singularity in Quantum Gravity, grqc/0407097.

[89] V. Husain, O. Winkler, On Singularity Resolution in Quantum Gravity, gr-qc/0312094, Phys.Rev. D69 (2004) 084016; Quantum resolution of black hole singularities, grqc/0410125.

[90] J. Brunnemann T. Thiemann On (Cosmological) Singularity Avoidance in Loop Quantum Gravity, gr-qc/0505032; Unboundedness of Triad - Like Operators in Loop Quantum Gravity, gr-qc/0505033

[91] S. Hofmann, O. Winkler, The Spectrum of Fluctuations in Inflationary Quantum Cosmology, astro-ph/0411124. 
[92] G. M. Hossain, Primordial Density Perturbation in Effective Loop Quantum Cosmology, gr-qc/0411012, Class.Quant.Grav. 22 (2005) 2511.

[93] A. Ashtekar, Martin Bojowald, Black hole evaporation: A paradigm, gr-qc/0504029.

[94] Abhay Ashtekar, Alejandro Corichi, Non-minimal couplings, quantum geometry and black hole entropy, gr-qc/0305082, Class.Quant.Grav. 20 (2003) 4473-4484

[95] S. Das, Parthasarathi Majumdar, Rajat K. Bhaduri, " General Logarithmic Corrections to Black Hole Entropy", Class.Quant.Grav. 19 (2002) 2355-2368; Saurya Das, hep-th/0207072, "Leading Log Corrections to Bekenstein-Hawking Entropy"

[96] Kirill Krasnov, "Quantum Geometry and Thermal Radiation from Black Holes", grqc/9710006, Class.Quant.Grav. 16 (1999) 563-578.

[97] M. Barreira, M. Carfora, C. Rovelli, "Physics with nonperturbative quantum gravity: radiation from a quantum black hole" gr-qc/9603064, Gen.Rel.Grav. 28 (1996) 12931299.

[98] J. D. Bekenstein, Black Holes And Entropy, Phys. Rev. D 7, 2333 (1973).

[99] S. W. Hawking, Particle Creation By Black Holes," Commun. Math. Phys. 43, 199 (1975).

[100] W. G. Unruh, Phys. Rev. D 14 (1976) 870.

[101] A. Ashtekar, C. Rovelli, L. Smolin, Weaving a classical metric with quantum threads," Phys. Rev. Lett. 69 (1992) 237.

[102] Luca Bombelli, Statistical geometry of random weave states, gr-qc/0101080

[103] A. Ashtekar, C. Rovelli, L. Smolin, Gravitons and loops, D 44 (1991) 1740-1755.

[104] J. Iwasaki, C. Rovelli, Gravitons as embroidery on the weave," Int. J. Mod. Phys. D 1 (1993) 533; Gravitons from loops: non-perturbative loop-space quantum gravity contains the graviton-physics approximation," Class. Quantum Grav. 11 (1994) 1653.

[105] M. Varadarajan, The graviton vacuum as a distributional state in kinematic Loop Quantum Gravity, gr-qc/0410120, Class.Quant.Grav. 22 (2005) 1207-1238; F. Conrady, Free vacuum for loop quantum gravity, gr-qc/0409036.

[106] H. Sahlmann, T. Thiemann, O. Winkler, Coherent States for Canonical Quantum General Relativity and the Infinite Tensor Product Extension, Nucl.Phys. B606 (2001) 401-440, gr-qc/0102038; T. Thiemann, Complexifier Coherent States for Quantum General Relativity, gr-qc/0206037.

[107] Fotini Markopoulou,"Coarse graining in spin foam models", gr-qc/0203036; 
[108] Fotini Markopoulou, "An algebraic approach to coarse graining", hep-th/0006199.

[109] H. Kodama, Prog. Theor. Phys. 80, 1024(1988); Phys. Rev. D42(1990)2548.

[110] L. Smolin and C. Soo, The Chern-Simons Invariant as the Natural Time Variable for Classical and Quantum Cosmology, Nucl. Phys. B449 (1995) 289, gr-qc/9405015.

[111] L. N. Chang and C. Soo, Ashtekar's variables and the topological phase of quantum gravity, Proceedings of the XXth. Conference on Differential Geometric Methods in Physics, (Baruch College, New York, 1991), edited by S. Catto and A. Rocha (World Scientific, 1992); Phys. Rev. D46 (1992) 4257; C. Soo and L. N. Chang, Int. J. Mod. Phys. D3 (1994) 529.

[112] C. Soo, Wave function of the Universe and Chern-Simons Perturbation Theory, grqc/0109046.

[113] A. Mikovic, Quantum gravity vacuum and invariants of embedded spin networks, grqc/0301047.

[114] R. Jackiw, Topological Investigations In Quantized Gauge Theories, p. 258, exercise 3.7, in S. B. Treiman et. al. Current Algebra And Anomalies (World Scientific, 1985).

[115] E. Witten, A note on the Chern-Simons and Kodama wavefunctions, arXiv:grqc/0306083.

[116] L. Friedel and L. Smolin, The linearizationof the Kodama state, hep-th/0310224.

[117] L. Freidel, J. Kowalski-Glikman, L. Smolin,2+1 gravity and Doubly Special Relativity, hep-th/0307085.

[118] G. Amelino-Camelia, L. Smolin, A. Starodubtsev,Quantum symmetry, the cosmological constant and Planck scale phenomenology,hep-th/0306134

[119] S. Alexander, J. Malecki, L. Smolin, Quantum Gravity and Inflation, hep-th/0309045

[120] M. Bojowald, G. Date, A non-chaotic quantum Bianchi IX universe and the quantum structure of classical singularities, gr-qc/0311003.

[121] S. Alexander, A Quantum Gravitational Relaxation of The Cosmological Constant, hepth/0503146.

[122] J. Christian, Testing Quantum Gravity via Cosmogenic Neutrino Oscillations, grqc/0409077, Phys.Rev. D71 (2005) 024012.

[123] L. Smolin, Falsifiable predictions from semiclassical quantum gravity, hep-th/0501091.

[124] A. Starodubtsev, String theory in a vertex operator representation: a simple model for testing loop quantum gravity, gr-qc/0201089. 
[125] T. Thiemann, The LQG - String: Loop Quantum Gravity Quantization of String Theory I. Flat Target Space, hep-th/0401172; D. Bahns, The invariant charges of the Nambu-Goto String and Canonical Quantization, hep-th/0403108, J.Math.Phys. 45 (2004) 4640-4660.

[126] R. Helling, G. Policastro String quantization: Fock vs. LQG Representations, hepth/0409182.

[127] O. Dreyer, Background Independent Quantum Field Theory and the Cosmological Constant Problem, hep-th/0409048.

[128] L. Smolin Some background for background independence, to appear.

[129] M. H. Ansari, L. Smolin, Self-organized criticality in quantum gravity, hep-th/0412307.

[130] A. Perez, C. Rovelli, Physical effects of the Immirzi parameter, gr-qc/0505081.

[131] Viqar Husain, "Observables for spacetimes with two Killing field symmetries", grqc/9402019,Phys.Rev. D50 (1994) 6207-6216; V. Husain and L. Smolin, "Exactly Solvable Quantum Cosmologies From Two Killing Field spacetimes", Nucl.Phys. B327 (1989) 205.

[132] M. Atiyah, Topological quantum field theory Publ. Math. IHES 68 (1989) 175; The Geometry and Physics of Knots, Lezion Lincee (Cambridge University Press, Cambridge,1990); G. Segal, Conformal field theory oxford preprint (1988).

[133] G. Horowitz, "Exactly Soluble Diffeomorphism Invariant Theories", Commun.Math.Phys. 125 (1989) 417; V. Husain, "Topological Quantum Mechanics", Phys.Rev. D43 (1991) 1803.

[134] R. Gianvittorio, R. Gambini and A. Trias, Phys. Rev. D38 (1988) 702; C. Rovelli and L. Smolin. Loop representation for lattice gauge theory,1990 Pittsburgh and Syracuse preprint; B. Bruegmann, Physical Review D 43 (1991) 566; J.M.A. Farrerons, Loop calculus for SU(3) on the lattice Phd. thesis, Universitat Autonoma de Barcelona (1990); R. Loll A new quantum representation for canonical gravity and SU(2) Yang-Mills theory, University of Bonn preprint, BONN-HE-90-02 (1990).

[135] N. Hitchin, The geometry of three-forms in six and seven dimensions, math.DG/0010054; Stable forms and special metrics, math.DG/0107101

[136] Robert Dijkgraaf, Sergei Gukov, Andrew Neitzke and Cumrun Vafa, Topological Mtheory as Unification of Form Theories of Gravity arXiv:hep-th/0411073

[137] L. Smolin, A quantization of topological $\mathcal{M}$ theory, hep-th/0503140

[138] F. Markopoulou and L. Smolin, Nonperturbative dynamics for abstract $(p, q)$ string networks, Phys.Rev. D58 (1998) 084033, hep-th/9712148. 
[139] L. Crane, A New Approach to the Geometrization of Matter,gr-qc/0110060.

[140] F, Markopoulou and L. Smolin, to appear.

[141] Sundance O.Bilson-Thompson, A topological model of composite preons, hep$\mathrm{ph} / 0503213$.

[142] F. Markopoulou and L. Smolin, Quantum Theory from Quantum Gravity, grqc/0311059

[143] G. Amelino-Camelia et al, Int.J.Mod.Phys.A12:607-624,1997; G. Amelino-Camelia et al Nature 393:763-765,1998; J. Ellis et al, Astrophys.J.535:139-151,2000; J. Ellis, N.E. Mavromatos and D. Nanopoulos, Phys.Rev.D63:124025,2001; ibidem astro$\mathrm{ph} / 0108295$.

[144] N. Hayashida, K. Honda, N. Inoue et. al. (The AGASA collaboration), Updated AGASA event list above $4 \times 10^{1} 9 \mathrm{eV}$, astro-ph/0008102, Astrophys.J. 522 (1999) 225; M. Takeda, N. Sakaki, K. Honda, (The AGASA collaboration), Energy determination in the Akeno Giant Air Shower Array experiment, astro-ph/0209422.

[145] G. Amelino-Camelia and T. Piran, Phys.Rev. D64 (2001) 036005.

[146] G. Amelino-Camelia, Nature 418 (2002) 34.

[147] J. Lukierski, H. Ruegg, A. Nowicki, and V.N. Tolstoy. q-deformation of Poincare algebra. Phys. Lett. B, 264,271:331,321, 1991; S. Majid. Braided groups and algebraic quantum field theories. Lett. Math. Phys., 22:167 176, 1991; Braided Momentum Structure of the q-Poincare Group, hep-th/9210141, J.Math.Phys. 34 (1993) 2045

[148] N.R. Bruno, G. Amelino-Camelia, J. Kowalski-Glikman, Phys.Lett.B522:133138,2001; J. Kowalski-Glikman and S. Nowak, hep-th/0203040;S. Judes, grqc/0205067; M. Visser, gr-qc/0205093; S. Judes, M. Visser, gr-qc/0205067; D. V. Ahluwalia and M. Kirchbach, qr-qc/0207004.

[149] J. Magueijo and L. Smolin, Phys.Rev.Lett. (88) 190403, 2002.

[150] J. Magueijo and L. Smolin, Generalized Lorentz invariance with an invariant energy scale, gr-qc/0207085, Phys.Rev. D67 (2003) 044017.

[151] Tomasz J. Konopka, Seth A. Major, “Observational Limits on Quantum Geometry Effects", New J.Phys. 4 (2002) 57. hep-ph/0201184; Ted Jacobson, Stefano Liberati, David Mattingly, "TeV Astrophysics Constraints on Planck Scale Lorentz Violation"; Threshold effects and Planck scale Lorentz violation: combined constraints from high energy astrophysics, hep-ph/0112207. 
[152] Subir Sarkar, " Possible astrophysical probes of quantum gravity", Mod.Phys.Lett. A17 (2002) 1025-1036, gr-qc/0204092.;David Mattingly, Modern tests of Lorentz invariance, gr-qc/0502097.

[153] S.D. Biller et al, Limits to Quantum Gravity Effects from Observations of TeV Flares in Active Galaxies, gr-qc/9810044; G. Amelino-Camelia, Improved limit on quantum-spacetime modifications of Lorentz symmetry from observations of gamma-ray blazars, gr-qc/0212002.

[154] G. Amelino-Camelia, John Ellis, N.E. Mavromatos, D.V. Nanopoulos, Subir Sarkar, Potential Sensitivity of Gamma-Ray Burster Observations to Wave Dispersion in Vacuo, astro-ph/9712103, Nature 393 (1998) 763-765; J.P. Norris, J.T. Bonnell, G.F. Marani, J.D. Scargle, GLAST, GRBs, and Quantum Gravity, astro-ph/9912136.

[155] J. Ellis, N.E. Mavromatos, D.V. Nanopoulos, A.S. Sakharov, Quantum-Gravity Analysis of Gamma-Ray Bursts using Wavelets, astro-ph/0210124

[156] T. Jacobson, S. Liberati, D. Mattingly, Lorentz violation and Crab synchrotron emission: a new constraint far beyond the Planck scale, astro-ph/0212190.

[157] Robert C. Myers, Maxim Pospelov, Experimental Challenges for Quantum Gravity, p$\mathrm{ph} / 0301124$.

[158] Reinaldo J. Gleiser, Carlos N. Kozameh, Astrophysical limits on quantum gravity motivated birefringence, gr-qc/0102093.

[159] Richard Lieu, Lloyd W. Hillman, Stringent limits on the existence of Planck time from stellar interferometry, astro-ph/0211402, astro-ph/0301184.

[160] Y. Jack Ng, W. A. Christiansen, H. van Dam, astro-ph/0302372

[161] Giovanni Amelino-Camelia, An interferometric gravitational wave detector as a quantum-gravity apparatus, gr-qc/9808029, Nature 398 (1999) 216-218.

[162] Robert H. Brandenberger and Jerome Martin On Signatures of Short Distance Physics in the Cosmic Microwave Background, hep-th/0202142, Int.J.Mod.Phys. A17 (2002) 36633680 .

[163] J. K. Webb, M. T. Murphy, V. V. Flambaum, S. J. Curran, Does the fine structure constant vary? A third quasar absorption sample consistent with varying alpha, astroph/0210531, ApSS, 283, 565, 200; M. T. Murphy, J. K. Webb, V. V. Flambaum, S. J. Curran, Does the fine structure constant vary? A detailed investigation into systematic effects, astro-ph/0210532, ApSS, 283, 577, 2003; John N. Bahcall, Charles L. Steinhardt, David Schlegel, Does the fine-structure constant vary with cosmological epoch?, astro-ph/0301507. 
[164] Laura Mersini, Mar Bastero-Gil, Panagiota Kanti, Relic Dark energy from TransPlanckian Regime, hep-ph/0101210, Phys.Rev. D64 (2001) 043508; Mar Bastero-Gil, Paul H. Frampton, Laura Mersini, Modified Dispersion Relations from Closed Strings in Toroidal Cosmology, Phys.Rev. D65 (2002) 106002, hep-th/0110167;

[165] J. Kogut and L. Susskind, Hamiltonian Formulation Of Wilson's Lattice Gauge Theories, Phys.Rev. D11 (1975) 395; W. Furmanski and A. Kowala, Yang-Mills Vacuum: An Attempt Of Lattice Loop Calculus, Journal-ref: Nucl.Phys. B291 (1987) 594.

[166] , H. Nicolai, K. Peeters, M. Zamaklar, Loop quantum gravity: an outside view hepth/0501114.

[167] L. Smolin, The classical limit and the form of the hamiltonian constraint in nonperturbative quantum gravity, gr-qc/9607034.

[168] J. Lewandowski, D. Marolf, Loop constraints: a habitat and their algebra," grqc/9710016; R. Gambini, J. Lewandowski, D. Marolf, J. Pullin, "On the consistency of the constraint algebra in spin-network quantum gravity," gr-qc/9710018.

[169] F. Markopoulou and L. Smolin, Gauge fixing in Causal Dynamical Triangulations, hepth/0409057.

[170] T. Konopka, Foliations and 2+1 Causal Dynamical Triangulation Models, hepth/0505004.

[171] M. H. Ansari, F. Markopoulou, A statistical formalism of Causal Dynamical Triangulations, hep-th/0505165.

[172] L. Crane in, Categorical Physics, hep-th/9301061; Topological Field theory as the key to quantum gravity, hep-th/9308126, in Knot theory and quantum gravity ed. J. Baez, (Oxford University Press); Clocks and Categories, is quantum gravity algebraic? J. Math. Phys. 36 (1995) 6180-6193, gr-qc/9504038.

[173] G. 'tHooft, Dimensional reduction in quantum gravity gr-qc/9310006.in Salanfestschrift ed. A. Alo, J. Ellis, S. Randjbar-Daemi (World Scienti c,1993).

[174] L. Susskind, The world as a hologram, hep-th/9409089, J. Math. Phys. 36 (1995) 6377 ; Strings, black holes and Lorentz contractions hepth/ 9308139; Phys. Rev. Lett. 71 (1993) 2367; Phys. Rev. D 49 (1994) 6606; D 50 (1994) 2700; L. Susskind and P. Gri n, Partons and black holes hep-th/9410306.

[175] T.Banks, "Cosmological Breaking of Supersymmetry?", hep-th/0007146.

[176] F. Markopoulou and L. Smolin, Holography in a quantum spacetime, hepth/9910146.

[177] L. Smolin, The strong and the weak holographic principles, hep-th/0003056. 